\title{
Weak vector boson production with many jets at the $\mathrm{LHC} \sqrt{s}=13 \mathrm{TeV}$
}

\author{
F. R. Anger, ${ }^{1}$ F. Febres Cordero, ${ }^{1}$ S. Höche, ${ }^{2}$ and D. Maître ${ }^{3}$ \\ ${ }^{1}$ Physikalisches Institut, Albert-Ludwigs-Universität Freiburg, D-79104 Freiburg, Germany \\ ${ }^{2}$ SLAC National Accelerator Laboratory, Menlo Park, California 94025, USA \\ ${ }^{3}$ Department of Physics, University of Durham, Durham DH1 3LE, United Kingdom
}

(Received 26 January 2018; published 23 May 2018)

\begin{abstract}
Signatures with an electroweak vector boson and many jets play a crucial role at the Large Hadron Collider, both in the measurement of Standard-Model parameters and in searches for new physics. Precise predictions for these multiscale processes are therefore indispensable. We present next-to-leading order QCD predictions for $W^{ \pm} / Z+$ jets at $\sqrt{s}=13 \mathrm{TeV}$, including up to five/four jets in the final state. All production channels are included, and leptonic decays of the vector bosons are considered at the amplitude level. We assess theoretical uncertainties arising from renormalization- and factorization-scale dependence by considering fixed-order dynamical scales based on the $H_{\mathrm{T}}$ variable as well as on the MiNLO procedure. We also explore uncertainties associated with different choices of parton-distribution functions. We provide event samples that can be explored through publicly available $n$-tuple sets, generated with BLACKHAT in combination with SHERPA.
\end{abstract}

DOI: 10.1103/PhysRevD.97.096010

\section{INTRODUCTION}

During the 7 and $8 \mathrm{TeV}$ runs of the Large Hadron Collider (LHC), the ATLAS and CMS experiments have scrutinized electroweak vector boson production in association with multiple light jets in great detail [1-4]. Recently, both collaborations presented first measurements $[5,6]$ of this class of processes at an energy of $13 \mathrm{TeV}$. These studies have shown the extent to which theoretical predictions produced both by dedicated calculations or by general Monte Carlo event generators can describe total rates and differential distributions for signatures that involve leptons, missing transverse energy, and many light jets. Such characterizations are of key importance given that searches for new physics carried out at hadron colliders target similar final states.

Precise calculations for vector-boson production with a single jet have reached a new era with the recent results at next-to-next-to-leading order (NNLO) QCD precision for $V+1$ jet [7] as well as many other refinements, which, for example, have been used to precisely describe backgrounds for dark matter searches [8]. At next-to-leading order (NLO) QCD, the state of the art is nowadays calculations for processes with four and five jets [9-11] in the final state. The matching to parton showers has been carried out for up

Published by the American Physical Society under the terms of the Creative Commons Attribution 4.0 International license. Further distribution of this work must maintain attribution to the author(s) and the published article's title, journal citation, and DOI. Funded by SCOAP ${ }^{3}$. to three jets in the final state [12], and multijet merging has been studied with up to two jets [13]. Next-to-leading order electroweak corrections have been computed for up to three jets [14] and combined with QCD merging [15].

In this article, we extend the set of NLO QCD calculations by dedicated predictions for the $13 \mathrm{TeV}$ LHC for $W+n$-jet and $Z+m$-jet production with $n \leq 5$ and $m \leq 4$. We employ the BLACKHAT library [16] for computing the required one-loop matrix elements. This library is based on on-shell and unitarity techniques (see, for example, [17]), which allows the extraction of loop amplitudes from simpler building blocks. It has been employed for many multilight-jet studies at hadron colliders [9-11,18-22] and has recently been extended to applications for highmultiplicity processes including heavy jets [23]. The calculation is performed with the help of the SHERPA package [24], used for integration over phase space as well as calculation of real corrections employing the Catani-Seymour dipole subtraction [25] as implemented in the matrix-element generator CoMIX [26]. We have stored our results and made them publicly available as Root [27] $n$-tuple files. This file format [28] contains all information necessary to compute the NLO fixed-order predictions, to change the running QCD coupling, renormalization, and factorization scales, as well as partondistribution functions (PDFs). We employ an extension [29] of the $n$-tuple file format which allows for extended reweighting procedures.

Due to its large center-of-mass energy, the LHC explores scales ranging from tens of GeV's to the multi- $\mathrm{TeV}$ regime. This large hierarchy of scales makes leading-order (LO) 
QCD predictions unreliable, as they tend to be very sensitive to the unphysical renormalization and factorization scales, and also because they can miss important initial-state partonic configurations. Next-to-leading order QCD calculations are less affected by scale choices and give a first reliable estimate of the theoretical uncertainty. ${ }^{1}$ We study in detail in this paper the theoretical uncertainties associated to our predictions that are related to scale sensitivity and PDF dependence. We explore in particular the scale dependence by using fixed-order dynamical scales based on the total partonic transverse energy as well as different variants of the MiNLO method [31]. In addition, we perform the conventional scale variation by constant factors around the central scales. A similar study was carried out recently for on-shell $t \bar{t}$ production in association with up to three light jets [32], and good agreement was found between NLO QCD results employing a similar set of dynamical scales. Our analysis is the first to compare results obtained with fixed-order scales and with the MiNLO method in processes with four or five light jets in the final state.

This paper is organized as follows. In Sec. II, we summarize our calculational setup, showing all kinematical information employed both at the level of producing $n$ tuple files and for the distributions studied in the rest of the paper. We also show in this section our implementation of the dynamical scales employed. In Sec. III, we present our results for total and differential cross sections and study their scale dependence and uncertainties associated with PDFs. In Sec. IV, we show a series of observable ratios that can help to reduce the theoretical uncertainties in multijet environments. The paper ends with our conclusions in Sec. V.

\section{BASIC SETUP}

We employ the SHERPA package [24] to manage the overall calculations. The required one-loop matrix elements are produced by the BLACKHAT library [16]. Born and realemission contributions are computed by the matrix-element generator Comix [26], which also provides the necessary Catani-Seymour subtraction terms [25]. More details of our computational setup can be found in Refs. [10,11]. We have included in all of our results all contributing subprocesses, confirming in particular that 8-quark finite contributions to the real part in $W^{ \pm}+5$-jet production are negligible [10].

We use the CT14 LO (CT14llo) and NLO (CT14nlo) PDFs [33] at the respective orders, including the corresponding definition of the strong coupling $\alpha_{s}$. We also employ the corresponding CT14nlo error set to explore PDF uncertainties and compare to predictions generated with the PDF error sets of Alekhin, Blümlein, and Moch

\footnotetext{
${ }^{1}$ A notable exception to this are processes with large accessible phase space and missing partonic channels at the next-to-leading order. This has been pointed out, for example, in [30].
}

(ABM) [34], Martin, Motylinski, Harland-Lang, and Thorne (MMHT) [35], and NNPDF 3.1 [36]. The leptonpair invariant mass follows a relativistic Breit-Wigner distribution, with $M_{W}=80.385 \mathrm{GeV}$ and $M_{Z}=91.1876 \mathrm{GeV}$, and the widths are given by $\Gamma_{Z}=2.4952 \mathrm{GeV}$ and $\Gamma_{W}=2.085 \mathrm{GeV}$. We employ a diagonal CKM matrix and use real values for the electroweak parameters.

All light quarks $(u, d, s, c$, and $b)$ are treated as massless. We do not include contributions from real or virtual top quarks, and we expect this to have a percentlevel effect on cross-sections [9,11,23,37]. The one-loop matrix elements for $V+4,5$-jets have been computed in the leading-color approximation [38], which we find to be precise at the level of $2 \%$ of the total cross section in lower jet-multiplicity calculations. Our results are quoted for a single lepton (pair) flavor. We treated both leptons as massless, an approximation that can be applied to the electron or muon families. Results presented are produced in fixed-order parton-level perturbation theory, and we do not apply any nonperturbative corrections to account for effects associated with underlying event or hadronization.

In the following subsections, we describe first the common setup to define the fiducial regions in which total and differential cross sections are computed. Second, we list the basic phase-space cuts applied at the level of producing the $n$-tuple sets and finally the dynamical scales that we employ to study the scale sensitivity of our results.

\section{A. Kinematical setup}

In our study, we consider the inclusive processes $p p \rightarrow V+n$ jets at the LHC with center-of-mass energy $\sqrt{s}=13 \mathrm{TeV}$, with $n \leq 5$ and $n \leq 4$ for $V=W^{ \pm}$and $Z$, respectively. We define jets using the anti- $k_{\mathrm{T}}$ algorithm [39] with $R=0.4$ and impose the kinematical cuts:

$$
p_{\mathrm{T}}^{\mathrm{jet}}>30 \mathrm{GeV}, \quad\left|\eta^{\mathrm{jet}}\right|<3 .
$$

We order the jets in $p_{\mathrm{T}}$ and label them according to their hardness. For all charged leptons we require

$$
p_{\mathrm{T}}^{l}>20 \mathrm{GeV}, \quad\left|\eta^{l}\right|<2.5 .
$$

For processes with $W^{ \pm}$bosons, we define its transverse mass by $M_{\mathrm{T}}^{W}=\sqrt{2 E_{\mathrm{T}}^{l} E_{\mathrm{T}}^{\nu}\left(1-\cos \left(\Delta \phi_{l \nu}\right)\right)}$. For these, we impose the additional cuts

$$
p_{\mathrm{T}}^{\nu}>20 \mathrm{GeV}, \quad M_{\mathrm{T}}^{W}>20 \mathrm{GeV} .
$$

Finally, for processes with a $Z$ boson we impose the following constraint on the invariant mass of its decay products:

$$
66 \mathrm{GeV}<M_{l^{+} l^{-}}<116 \mathrm{GeV} .
$$




\section{B. Kinematical setup for public $\boldsymbol{n}$-tuples}

We have saved intermediate results in publicly available Root-format [27] $n$-tuple files [28] in order to facilitate new studies of infrared-safe observables with different cuts, different scale choices, jet algorithms, or PDF sets. Our study uses an extension of the original file format which facilitates reweighting in the MiNLO procedure and enables extended reweighting procedures [29]. Due to the generation cuts applied to events stored in the $n$-tuple files, future studies should operate either with identical cuts or with tighter cuts and can operate with all listed jet recombination scenarios since the most inclusive one is chosen at the generation level.

We use the FASTJET package [40] to define jets according to the algorithms anti- $k_{\mathrm{T}}, k_{\mathrm{T}}$, and SISCONE [39,41,42] with jet parameter $R=0.4,0.5,0.6$, and 0.7. We set the recombination $f$-parameter for the SISCONE algorithm to 0.75 . We impose $p_{\mathrm{T}}^{\text {jet }}>30 \mathrm{GeV}$ on all jets and do not constrain their pseudorapidity.

For charged leptons, we impose $p_{\mathrm{T}}^{l}>20 \mathrm{GeV}$ and $\left|\eta^{l}\right|<3$, while for neutrinos we require $p_{\mathrm{T}}^{\nu}>10 \mathrm{GeV}$. For processes with a $Z$ boson, in which $\gamma^{*}$ contributions appear, we also constrain the associated lepton-pair invariant mass by $60 \mathrm{GeV}<M_{l^{+} l^{-}}<120 \mathrm{GeV}$.

\section{Dynamical scale choices}

We explore the renormalization and factorization scale dependence of the cross sections using a conventional variation of the central scale by factors $(1 / 2,1 / \sqrt{2}$, $1, \sqrt{2}, 2)$, keeping factorization and renormalization scales equal $^{2}$. In addition, we explore the sensitivity of the calculation to the functional form of the scale using on one hand conventional fixed-order scales based on the $H_{\mathrm{T}}$ variable and on the other hand the so-called MiNLO procedure [31]. In this subsection, we first define all the fixed-order scales employed, and second we present our variant of the MiNLO procedure, which we will label MiNLO'. Finally, we summarize the nomenclature used in this article to label all dynamical scales considered.

\section{Fixed-order scales}

We define the total partonic transverse energy variable $\hat{H}_{\mathrm{T}}^{\prime}$ according to

$$
\hat{H}_{\mathrm{T}}^{\prime}=\sum_{j} p_{\mathrm{T}}^{j}+E_{\mathrm{T}}^{V}
$$

\footnotetext{
${ }^{2}$ Based on a study of $W^{-}+1,2,3,4$-jet production, we have found that when sampling the $\left(\mu_{R}, \mu_{F}\right)$ plane over the values $[(1 / 2,1 / 2),(1 / 2,1),(1,1 / 2),(1,1),(1,2),(2,1),(2,2)]$, the estimated scale sensitivity is similar to what we find with the correlated variations.
}

where the sum runs over all final-state partons, and $E_{\mathrm{T}}^{V} \equiv$ $\sqrt{M_{V}^{2}+\left(p_{\mathrm{T}}^{V}\right)^{2}}$ is the transverse energy of the vector boson ( $V$ either $W$ or $Z$ ). The scale $\mu_{0}=\hat{H}_{\mathrm{T}}^{\prime} / 2$ has proven to be a sensible choice as it tends to reduce the shape changes and global size of quantum corrections when going from leading to next-to-leading order (see, for example, $[10,11,22])$. In general, NLO corrections are less sensitive to the choices of scale, as long as the scale reflects the hardness of the Born process [19].

We introduce an additional scale, designed to match the invariant mass of the lepton pair in kinematic configurations with very small hadronic transverse energy and the transverse momentum of the hardest QCD jet in processes of the dijet type. This scale is denoted as

$$
\hat{S}_{\mathrm{T}}=\frac{1}{2} \sum_{j} p_{\mathrm{T}}^{j}+E_{\mathrm{T}}^{V}
$$

In Sec. II C 3, we give the standard notation used throughout this article to refer to the different scales employed.

\section{MiNLO'}

The second type of dynamical scale considered in this study, schematically denoted as MiNLO', is based on the MiNLO reweighting procedure proposed in Ref. [31], which is inspired by the next-to-leading log (NLL) branching formalism in [41]. It builds on an event-by-event identification of the most likely branching history leading to the full $V+n$-parton final state using a $k_{T}$-type clustering algorithm. No-branching probabilities in the form of NLL Sudakov form factors are assigned to the intermediate "partons" in the branching tree to reflect the fact that no radiation above a resolution scale, given by the lowest nodal $k_{T}$ value in the clustering, should occur. The strong coupling associated with each node in the branching tree is evaluated at the respective transverse momentum, following [43]. This method can also be interpreted as a generalization of the Catani, Krauss, Kuhn, and Webber (CKKW) procedure [44] to NLO QCD calculations but without the possibility to further develop the intrajet real radiation pattern through resolved emissions. In particular, the MiNLO method accounts for the resummation of large logarithmic corrections associated with very disparate scales in high-energy collisions using the known universal factorization properties of the cross section in the collinear limit.

In order to reflect the nature of QCD interactions, only $1 \rightarrow 2$ branchings consistent with elementary interaction vertices are allowed in our MiNLO' procedure. In addition, we require the branching history to be ordered, which implies that we terminate the clustering as soon as an inverted scale hierarchy is encountered. In this case, by default we set the scale of the remaining $V+m$-parton (with $m<n$ ) "core" interaction, $\mu_{\text {core }}$, to $\hat{H}_{\mathrm{T}}^{\prime} / 2$ (or when 
explicitly stated, to $\hat{S}_{\mathrm{T}}$ ). This biases the scale choice for events with many hard scales toward $\hat{H}_{\mathrm{T}}^{\prime} / 2\left(\hat{S}_{\mathrm{T}}\right)$, an effect that will be further discussed in Sec. III. Note in particular that at very high energies there may be configurations where no clustering can be performed at all, for example a $V+2$ jet event with $p_{T, j 1} \approx p_{T, j 2} \gg m_{T, W}$ or a $V+5$ jet event where $p_{T, j 1} \approx p_{T, j 2} \approx \ldots \approx p_{T, j 5}$ and $y_{j 1} \ll y_{j 2} \ll \ldots \ll y_{j 5}$. The large logarithms associated with such configurations cannot be resummed in QCD collinear factorization and are therefore not amenable to a treatment in the coherent branching formalism on which the MiNLO method is based. This is a considerable source of uncertainty because of the large available phase space at the LHC.

In general, the clustering procedure for a leading-order process of $\mathcal{O}\left(\alpha_{s}^{N}\right)$ will yield a branching history with $M \leq N$ ordered nodal scales $q_{1} \ldots q_{M}$ and a core interaction of $\mathcal{O}\left(\alpha_{s}^{N-M}\right)$ with scale $\mu_{\text {core }}>q_{M}$. We then set the global renormalization scale $\mu_{R}$ to the geometric mean $\mu_{R}^{N}=\mu_{\text {core }}^{N-M} \prod_{i=1}^{M} q_{i}$.

Both intermediate lines (connecting branching nodes $i$ and $j$ ) and external lines are dressed with Sudakov form factors to reflect the no-branching probability. External lines connected to the $i$-th branching are multiplied by a factor $\Delta_{a}\left(q_{\min }, q_{i}\right)$, where the lowest branching scale $q_{1}=q_{\min }$ is identified as the resolution scale. Intermediate lines connecting nodes $j<i$ are dressed by factors $\Delta_{a}\left(q_{\min }, q_{i}\right) / \Delta_{a}\left(q_{\min }, q_{j}\right)$. Internal lines connected to the primary process are assigned form factors between their respective scales and $\mu_{\text {core }}$. The factorization scale $\mu_{F}$ used in the evaluation of the PDFs is set to the lowest scale, $\mu_{F}=q_{1}$.

In our MiNLO' procedure, we use a physical definition of the Sudakov form factors, which is given by

$$
\begin{aligned}
\Delta_{a}\left(Q_{0}, Q\right)= & \exp \left\{-\int_{Q_{0}}^{Q} \frac{\mathrm{d} q}{q} \frac{\alpha_{s}(q)}{\pi} \sum_{b=q, g} \int_{0}^{1-q / Q} \mathrm{~d} z\right. \\
& \left.\times\left(z P_{a b}(z)+\delta_{a b} \frac{\alpha_{s}(q)}{2 \pi} \frac{2 C_{a}}{1-z} K\right)\right\}
\end{aligned}
$$

where [45]

$$
K=\left(\frac{67}{18}-\frac{\pi^{2}}{6}\right) C_{A}-\frac{10}{9} T_{R} n_{f}
$$

and $a=g, q$ corresponds to massless gluons and quarks, respectively. Equation (2.7) does not exceed unity and can therefore be interpreted as a no-branching probability between the scales $Q_{0}$ and $Q$, while maintaining the correct limiting behavior for $Q_{0} \ll Q$. The above definition is easily obtained from the known NLL expressions of [46] by employing the following symmetry of the LO splitting functions

$$
\begin{aligned}
\sum_{b=q, g} \int_{0}^{1-\varepsilon} d z z P_{q b}(z)= & \int_{\varepsilon}^{1-\varepsilon} d z P_{q q}(z)+\mathcal{O}(\varepsilon) \\
\sum_{b=q, g} \int_{0}^{1-\varepsilon} d z z P_{g b}(z)= & \int_{\varepsilon}^{1-\varepsilon} d z\left[\frac{1}{2} P_{g g}(z)+n_{f} P_{g q}(z)\right] \\
& +\mathcal{O}(\varepsilon) .
\end{aligned}
$$

Following standard practice, we include next-to-leading logarithms proportional to the two-loop cusp anomalous dimension [45].

The MiNLO method requires some extra specifications [31]. Virtual corrections and integrated IR-subtraction terms are treated identically to the leading order case. Real-emission events have branching histories with $M+$ $1 \leq N+1$ ordered branchings, but they are treated as Born-like $M$-parton events for the purpose of scale definition. This is achieved by discarding the softest branching, i.e., if the $M+1$ step branching history is given by $q_{0}<q_{1}<\ldots<q_{K}$, we set the resolution scale to $q_{1}$. Consequently, the softest emission at NLO (with scale $q_{0}$ ) is neither dressed with Sudakov factors nor does it enter the definitions of $\mu_{R}$ and $\mu_{F}$. In order to retain NLO accuracy of the full calculation, the Born configuration receives correction terms that are proportional to the firstorder expansion of the Sudakov factors, Eq. (2.7). Note that in this case the scale of the strong coupling is set to $\mu_{R}$ and that the two-loop cusp term is neglected, as it contributes at $\mathcal{O}\left(\alpha_{s}^{2}\right)$. The value of the additional strong coupling at NLO (the $N+1$-th power) appearing in both real and virtual corrections is set to the average of all other values of $\alpha_{s}$, i.e., $N \alpha_{s}^{(N+1)}=(N-M) \alpha_{s}\left(\mu_{\text {core }}\right)+\sum_{i=1}^{M} \alpha_{s}\left(q_{i}\right)$. Conventional scale uncertainties associated with the MiNLO method are estimated using variations of $\mu_{R}$ and $\mu_{F}$ by constant factors of two. The scale of the strong coupling in the Sudakov form factors remains fixed at the integration variable, $q$, while it is varied in all other parts of the calculation, including the MiNLO counterterms used to subtract the $\mathcal{O}\left(\alpha_{s}\right)$ expansion of the Sudakov factors. Factorization scale variations in the MiNLO' procedure have been discussed extensively in [32]. We perform them in the same manner, i.e., we set $q_{1}$ equal to $\mu_{F}$.

\section{Nomenclature for dynamical scales explored}

Throughout this paper, we set renormalization and factorization scales equal $\mu_{R}=\mu_{F}=\mu_{0}$, and for scaledependence bands, we make variations by factors of $(1 / 2,1 / \sqrt{2}, 1, \sqrt{2}, 2)$. Our results labeled LO and NLO use the central scale $\mu_{0}=\hat{H}_{\mathrm{T}}^{\prime} / 2$ by default, where $\hat{H}_{\mathrm{T}}^{\prime}$ is defined in Eq. (2.5). When necessary, to distinguish the usage of the fixed-order scales defined in Sec. II C 1, we write "(N)LO $\hat{H}_{\mathrm{T}}^{\prime} / 2$ " or "(N)LO $\hat{S}_{\mathrm{T}}$ ", where $\hat{S}_{\mathrm{T}}$ is defined in Eq. (2.6).

In our MiNLO' procedure described in Sec. II C 2, the default core scale is $\mu_{\text {core }}=\hat{H}_{\mathrm{T}}^{\prime} / 2$. When considering 
variations of this choice, we explicitly write ' $\mathrm{Mi}(\mathrm{N}) \mathrm{LO}$ ' $\hat{H}_{\mathrm{T}}^{\prime} / 2$ " or "Mi (N) LO' $\hat{S}_{\mathrm{T}}$ ".

We also compare to the original formulation of the MiNLO method [31] for processes with fewer than three jets in the final state. Compared to our implementation, this variant uses the Sudakov factors of [46], and unordered clustering histories are treated in a different manner. Following the previous naming convention, we label those results as "Mi (N) LO $\hat{H}_{\mathrm{T}}^{\prime} / 2$ " or "Mi (N) LO $\hat{S}_{\mathrm{T}}$ " depending on the choice of core scale $\mu_{\text {core }}$ employed.

\section{RESULTS FOR $V+$ Jets PRODUCTION}

\section{A. Total cross sections}

In Tables I, II, and III, we present total cross sections for the production of a weak vector boson $V$ in association with up to 5 jets for $V=W^{+}$and $W^{-}$and with up to 4 jets for $V=Z$. Results with central scale $\hat{H}_{\mathrm{T}}^{\prime} / 2$ and MiNLO' are included (see Sec. II C 3 for the nomenclature that we use for the different dynamical scales considered). We also show in Table IV the jet production ratios $[22,47]$ for all of the vector bosons, that is, the ratios of the total cross sections for the production for $V+n$ jets to the production of $V+(n-1)$ jets.

We observe that $\mathrm{LO}$ cross sections for the scale choice $\mu_{0}=\hat{H}_{\mathrm{T}}^{\prime} / 2$ have a monotonic increase of scale dependence from about $20 \%$ for $V+2$ jet up to $50 \%$ for $V+5$ jets. We notice that the LO scale dependence for $V+1$ jet, which appears at around 4\%, is not representative of the associated theoretical uncertainties, in particular due to kinematical constraints that are released at NLO. That is, at LO the $p_{\mathrm{T}}$ of the vector boson matches the one from the unique jet. Real contributions at NLO release this constraint, producing a soft enhancement that tends to produce large corrections $[19,30,48]$.

The central scale choice for the dynamical scale $\mu_{0}=\hat{H}_{\mathrm{T}}^{\prime} / 2$ falls near the plateau of the NLO scale dependence. This makes the uncertainty estimates based on lower/upper values of the cross sections seem slightly small. If we quote the absolute deviations with respect to this value, then we can estimate NLO scale sensitivity at the order of $10 \%$ (running from about $6 \%$ to $16 \%$, depending on multiplicity).

TABLE I. LO and NLO QCD results for inclusive $W^{+}+1,2,3,4$, 5-jet total cross sections (in $p b$ ). Results are quoted for the two dynamical scales, $\hat{H}_{\mathrm{T}}^{\prime} / 2$ and MiNLO', as well as their ratio. Details of the calculation are given in Sec. II A. The conventional scale dependence is determined by varying $\mu_{R}$ and $\mu_{F}$ by factors of 2 and $\sqrt{2}$ up and down. The number in parenthesis gives the statistical error from the numerical integration.

\begin{tabular}{lcccccc}
\hline \hline jets & $W^{+} \mathrm{LO}$ & $W^{+} \mathrm{NLO}$ & $W^{+} \mathrm{MiLO}^{\prime}$ & $W^{+} \mathrm{MiNLO}^{\prime}$ & $\mathrm{MiLO}^{\prime} / \mathrm{LO}$ & $\mathrm{MiNLO}^{\prime} / \mathrm{NLO}$ \\
\hline 1 & $588.49(33)_{-27.07}^{+23.77}$ & $764.9(16)_{-27.1}^{+37.8}$ & $591.50(35)_{-25.36}^{+20.70}$ & $799.1(18)_{-35.1}^{+49.9}$ & $1.005(1)$ & $1.045(3)$ \\
2 & $197.23(27)_{-34.42}^{+44.64}$ & $197.78(66)_{-7.82}^{+1.80}$ & $205.01(28)_{-36.46}^{+46.95}$ & $211.44(78)_{-12.07}^{+9.50}$ & $1.039(2)$ & $1.069(5)$ \\
3 & $57.07(10)_{-15.23}^{+22.82}$ & $49.54(27)_{-3.13}^{+0.00}$ & $59.09(11)_{-16.89}^{+26.02}$ & $52.32(41)_{-3.95}^{+0.19}$ & $1.035(3)$ & $1.056(10)$ \\
4 & $16.408(50)_{-5.566}^{+9.344}$ & $12.14(22)_{-1.59}^{+0.00}$ & $17.287(56)_{-6.516}^{+11.768}$ & $12.78(24)_{-2.87}^{+0.00}$ & $1.054(5)$ & $1.053(27)$ \\
5 & $4.579(45)_{-1.829}^{+3.399}$ & $3.06(14)_{-0.72}^{+0.00}$ & $4.908(56)_{-2.233}^{+4.691}$ & $3.21(14)_{-0.52}^{+0.00}$ & $1.072(16)$ & $1.049(66)$ \\
\hline \hline
\end{tabular}

TABLE II. As in Table I but for inclusive $W^{-}+1,2,3,4,5$-jet total cross sections.

\begin{tabular}{lcccccc}
\hline \hline jets & $W^{-} \mathrm{LO}$ & $W^{-} \mathrm{NLO}$ & $W^{-} \mathrm{MiLO}^{\prime}$ & $W^{-} \mathrm{MiNLO}^{\prime}$ & $\mathrm{MiLO}^{\prime} / \mathrm{LO}$ & $\mathrm{MiNLO}^{\prime} / \mathrm{NLO}$ \\
\hline 1 & $446.68(22)_{-20.29}^{+17.48}$ & $582.0(11)_{-18.6}^{+25.6}$ & $448.82(23)_{-18.92}^{+15.07}$ & $608.2(12)_{-24.5}^{+34.6}$ & $1.005(1)$ & $1.045(3)$ \\
2 & $141.67(14)_{-24.67}^{+31.89}$ & $144.53(39)_{-5.46}^{+1.03}$ & $147.40(15)_{-26.08}^{+33.40}$ & $154.11(46)_{-8.40}^{+5.91}$ & $1.040(1)$ & $1.066(4)$ \\
3 & $39.029(55)_{-10.448}^{+15.653}$ & $34.34(16)_{-2.10}^{+0.00}$ & $40.889(61)_{-11.70}^{+17.99}$ & $36.20(22)_{-2.64}^{+0.03}$ & $1.048(2)$ & $1.054(8)$ \\
4 & $10.513(23)_{-3.585}^{+6.035}$ & $8.22(13)_{-0.86}^{+0.00}$ & $11.399(27)_{-4.734}^{+7.797}$ & $8.85(19)_{-1.85}^{+0.00}$ & $1.084(3)$ & $1.077(29)$ \\
5 & $2.747(12)_{-1.103}^{+2.059}$ & $1.971(56)_{-0.298}^{+0.004}$ & $3.063(14)_{-1.400}^{+2.949}$ & $2.105(59)_{-0.788}^{+0.000}$ & $1.115(7)$ & $1.068(43)$ \\
\hline \hline
\end{tabular}

TABLE III. As in Table I but for inclusive $Z+1,2,3$, 4-jet total cross sections.

\begin{tabular}{|c|c|c|c|c|c|c|}
\hline jets & $Z \mathrm{LO}$ & $Z \mathrm{NLO}$ & Z MiLO' & Z MiNLO' & MiLO' / LO & MiNLO' / NLO \\
\hline 1 & $112.264(60)_{-4.876}^{+4.121}$ & $142.79(15)_{-3.70}^{+5.12}$ & $112.615(43)_{-4.448}^{+3.390}$ & $148.48(17)_{-5.04}^{+7.21}$ & $1.003(1)$ & $1.040(2)$ \\
\hline 2 & $36.140(38)_{-6.178}^{+7.931}$ & $36.811(65)_{-1.339}^{+0.228}$ & $36.780(28)_{-6.382}^{+8.076}$ & $38.962(68)_{-2.147}^{+1.555}$ & $1.018(1)$ & $1.058(3)$ \\
\hline 3 & $10.4844(76)_{-2.7702}^{+4.1227}$ & $9.175(44)_{-0.578}^{+0.000}$ & $11.1242(87)_{-3.166}^{+4.847}$ & $9.612(50)_{-0.617}^{+0.000}$ & $1.061(1)$ & $1.048(7)$ \\
\hline 4 & $2.9597(37)_{-0.9989}^{+1.6698}$ & $2.331(29)_{-0.246}^{+0.000}$ & $3.3050(43)_{-1.247}^{+2.248}$ & $2.439(37)_{-0.668}^{+0.000}$ & $1.117(2)$ & $1.046(21)$ \\
\hline
\end{tabular}


TABLE IV. LO, NLO, and MiNLO' QCD jet production ratios for $W^{ \pm}$as well as $Z / \gamma^{*}$. The ratio is taken for a given process to that with one fewer jet. The setup is specified in Sec. II A. The number in parenthesis next to the ratio gives the corresponding statistical integration error.

\begin{tabular}{|c|c|c|c|c|c|c|c|c|c|}
\hline \multirow[b]{2}{*}{$n$} & \multicolumn{3}{|c|}{$W^{+} n /(n-1)$} & \multicolumn{3}{|c|}{$W^{-} n /(n-1)$} & \multicolumn{3}{|c|}{$Z n /(n-1)$} \\
\hline & LO & NLO & MiNLO' & LO & NLO & MiNLO' & LO & NLO & MiNLO' \\
\hline 2 & $0.3351(5)$ & $0.259(1)$ & $0.265(1)$ & $0.3172(4)$ & $0.248(1)$ & $0.253(1)$ & $0.3219(4)$ & $0.2578(5)$ & $0.2624(5)$ \\
\hline 3 & $0.2894(6)$ & $0.250(2)$ & $0.247(2)$ & $0.2755(5)$ & $0.238(1)$ & $0.235(2)$ & $0.2901(4)$ & $0.249(1)$ & $0.247(1)$ \\
\hline 4 & $0.288(1)$ & $0.245(5)$ & $0.244(5)$ & $0.2694(7)$ & $0.239(4)$ & $0.244(5)$ & $0.2823(4)$ & $0.254(3)$ & $0.254(4)$ \\
\hline 5 & $0.279(3)$ & $0.252(12)$ & $0.251(11)$ & $0.261(1)$ & $0.240(8)$ & $0.238(8)$ & $\ldots$ & $\ldots$ & $\ldots$ \\
\hline
\end{tabular}

We find similar total cross sections with MiLO' to the corresponding LO results. Nevertheless, the absolute predictions are larger for $\mathrm{MiLO}^{\prime}$, and the excess increases slightly with multiplicity. In the case of MiNLO' results, the central predictions agree well with the NLO results. Moreover, their ratio is rather stable as a function of the jet multiplicity.

In Figs. 1-3, we display total cross sections and scale variations for all scales considered in this analysis. It can be seen that in particular the MiLO' and MiNLO' predictions exhibit considerable variation, both in their central value and in the associated conventional scale uncertainty. This can be traced back to the procedure for the identification of ordered clustering hierarchies, and the associated value of the scale of the core interaction, $\mu_{\text {core }}$ (cf. Sec. II C 2). Since we require the branching history to be ordered, we must terminate the clustering as soon as an inverted scale hierarchy is encountered.
This biases the scale choice for events with many hard scales toward $\mu_{\text {core }}$, and therefore, the precise definition of $\mu_{\text {core }}$ plays a significant role [49]. The choice of $\hat{S}_{\mathrm{T}}$ increases $\mu_{\text {core }}$ on average, thus permitting more clusterings in high-multiplicity final states, and therefore inducing more associated Sudakov form factors. On average, this reduces both the central value of the prediction and the related scale uncertainty.

Jet-production ratios have been shown to be a good handle for precision tests of QCD. In these ratios, many uncertainties associated with scale sensitivity and PDF dependence cancel to a large extent. Also, in experimental analyses, systematic uncertainties associated with luminosity measurements are canceled. In Table IV, we observe a remarkable stability of these ratios at NLO for both scale choices $\hat{H}_{\mathrm{T}}^{\prime} / 2$ and MiNLO', all of them falling around a value of 0.25 . This universality is present for NLO results in $V+$ jet even though the corresponding LO results

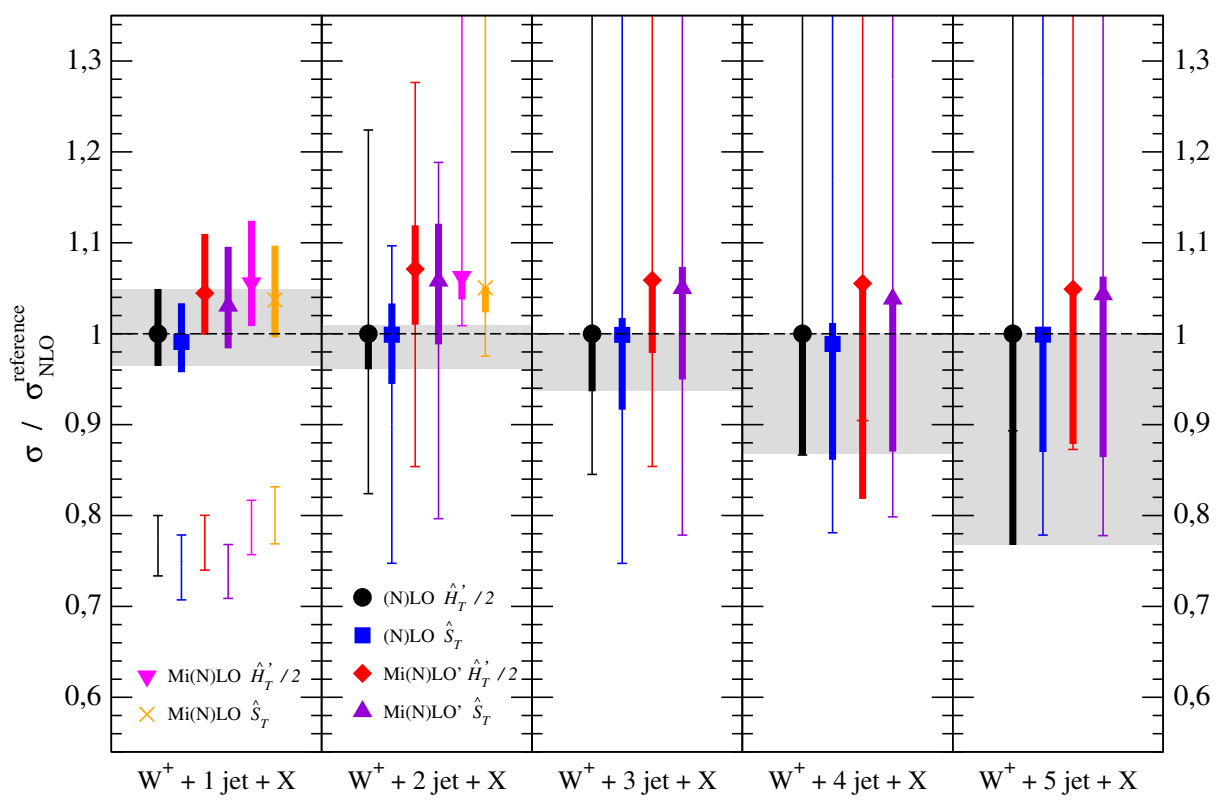

FIG. 1. Scale dependence of total cross sections for inclusive $W^{+}+1,2,3,4,5$-jet production considering all scales named in Sec. II C 3. Each scale is presented with a corresponding symbol (and color) specified in the label of the plot. The thin lines represent corresponding LO scale-dependence bands (in certain cases extending outside of the range plotted), while the thick lines represent NLO bands. All results have been normalized to the corresponding NLO $\hat{H}_{\mathrm{T}}^{\prime} / 2$ result at each jet multiplicity. The gray band shows the NLO $\hat{H}_{\mathrm{T}}^{\prime} / 2$ scale-dependence band. 


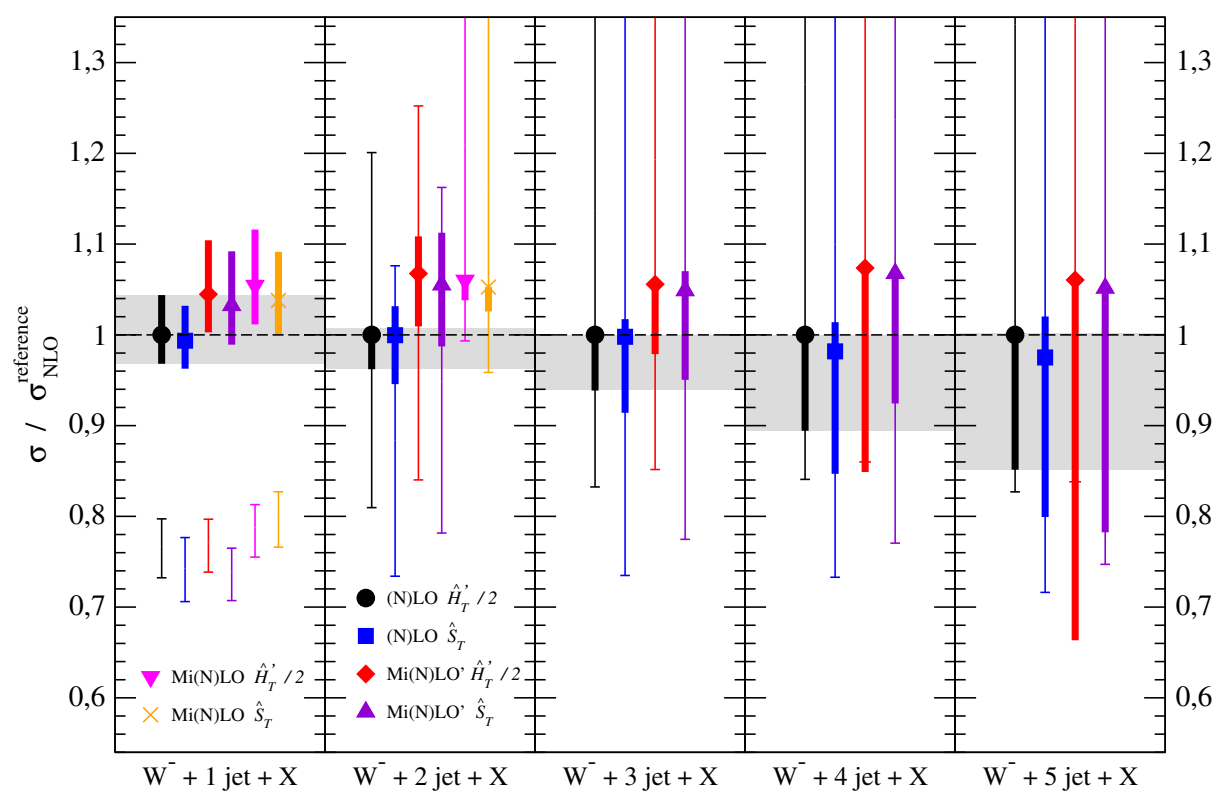

FIG. 2. As in Fig. 1 but for inclusive $W^{-}$production in association with jets. See Sec. II C 3 for details of the dynamical scales considered.

deviate considerably. The universality of the jet production ratios can be very helpful for tests of the standard model (SM) and can even be exploited to make extrapolations of total and differential cross sections to large jet-multiplicity processes [22].

\section{B. Scale dependence}

Figure 4 displays the dependence of total cross sections in $W^{-}$production in association with up to 5 jets on the renormalization and factorization scales. Results for the production of $W^{+}$or $Z$ in association with jets are similar.

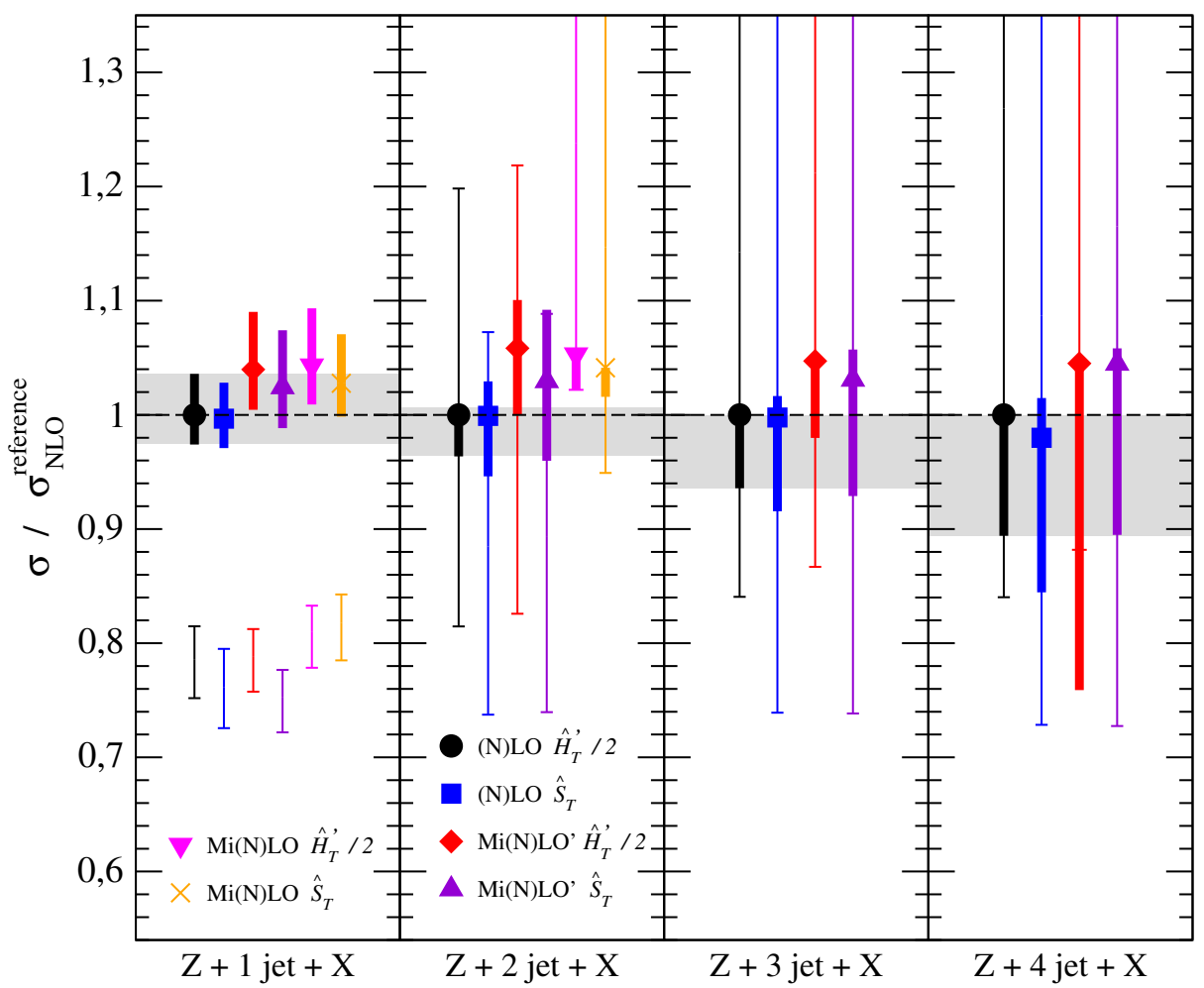

FIG. 3. As in Fig. 1 but for inclusive $Z$ production in association with jets. See Sec. II C 3 for details of the dynamical scales considered. 


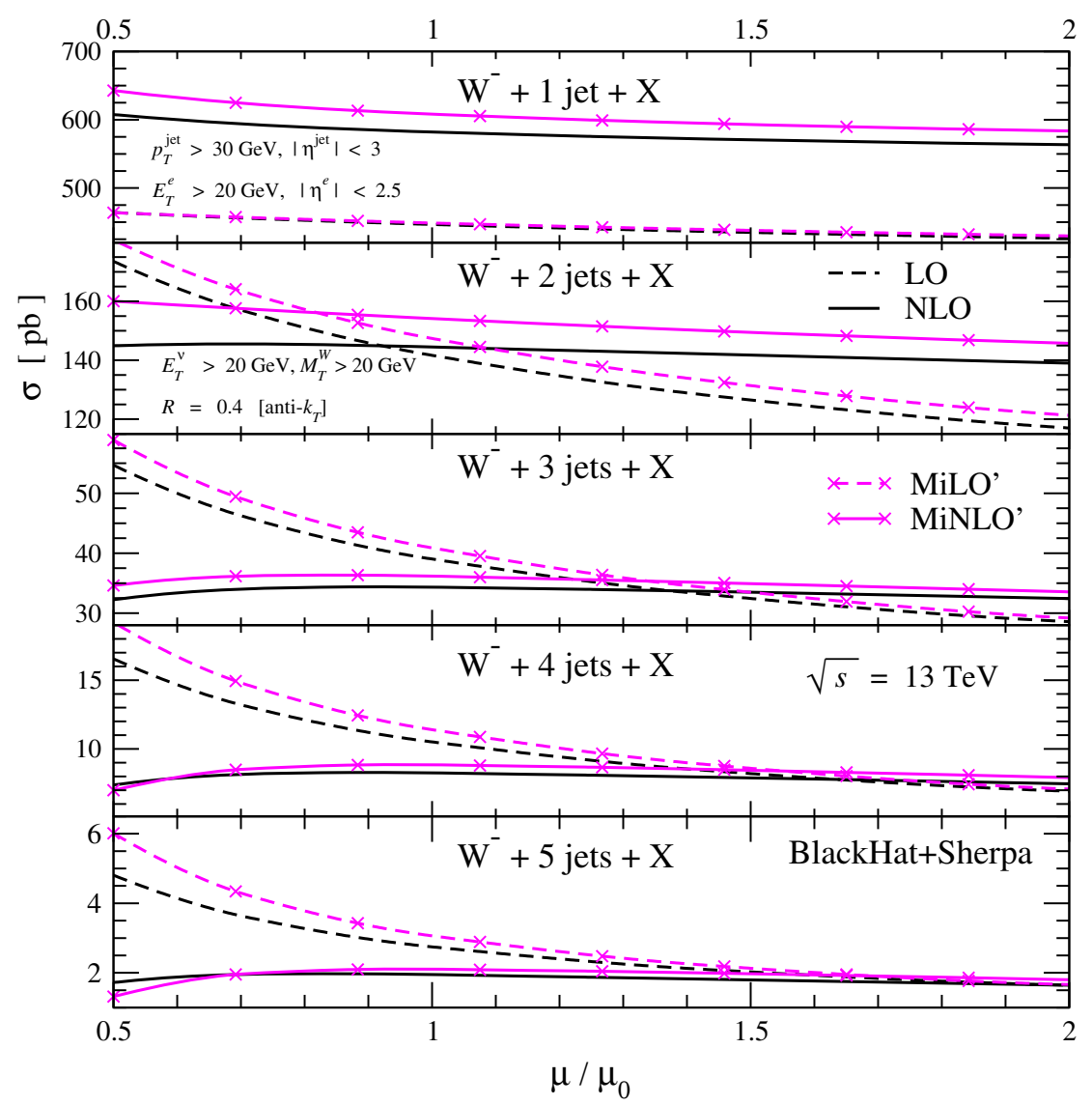

FIG. 4. Renormalization- and factorization-scale dependence of total cross sections for inclusive $W^{-}+1,2,3,4,5$-jet production. For each multiplicity, we show the dependence of predictions at LO as dashed (black) lines and at NLO as solid (black) lines with $\mu_{0}=\mu_{\mathrm{r}}=\mu_{\mathrm{f}}=\hat{H}_{\mathrm{T}}^{\prime} / 2$, while predictions for MiLO' are shown as dashed-crossed (magenta) lines and those for MiNLO' as solid-crossed (magenta) lines.

Although $\mathrm{LO}$ and MiLO' results are very sensitive to these scales, we find a remarkable stability for NLO and MiNLO' results. Note that the central prediction lies at the plateau of the NLO curves for $W^{-}+3,4$ and 5 jets, thus minimizing the scale variations (for a discussion of this effect, see [19]).

LO results obtained with the two scale choices appear as largely consistent, concerning both normalization and scale sensitivity. MiLO' results are slightly larger compared to LO results obtained with $\hat{H}_{\mathrm{T}}^{\prime} / 2$. This indicates that the small average renormalization scales for large jet multiplicity in $\mathrm{MiLO}^{\prime}$ and the correspondingly large strong couplings are not entirely compensated by the suppression from Sudakov form factors. The LO results obtained with the two scale choices differ in their variation. At low multiplicity, the scale uncertainty associated with LO and MiLO' results are comparable, but for increased multiplicity, the MiLO' results exhibit larger scale variations.

We observe that the NLO results obtained with both dynamical scales are mostly consistent. For multiplicities with more than 2 jets, the differences between the two scale choices lie within the respective factor-two scale variations.
In the case of $W+2$-jet production, an approximately $15 \%$ discrepancy appears, which can be taken as an estimate for the total scale uncertainty associated with the prediction. Furthermore, we observe that with increasing multiplicity, the bands associated with the uncertainty of the respective scale choice seem to behave differently between $\hat{H}_{\mathrm{T}}^{\prime} / 2$ and the MiNLO' scheme. In general, the scale uncertainty obtained by factor-two variations grows with multiplicity, e.g., for $\hat{H}_{\mathrm{T}}^{\prime} / 2$, from around $4.5 \%$ for $W^{-}+2 j$ to about $15 \%$ for $W^{-}+5 j$ production. It is now interesting to observe that this increase in scale uncertainty is more pronounced for the MiNLO' scale choice, where the uncertainty grows from around $6.5 \%\left(W^{-}+2 j\right)$ to around $35 \%\left(W^{-}+5 j\right)$.

\section{Differential distributions}

In this section, we analyze several differential distributions and the impact that higher-order corrections have on fixed-order predictions over phase space. In what follows, we show results only for the $W^{-}$weak vector boson, as in general the structure of QCD corrections is very similar 


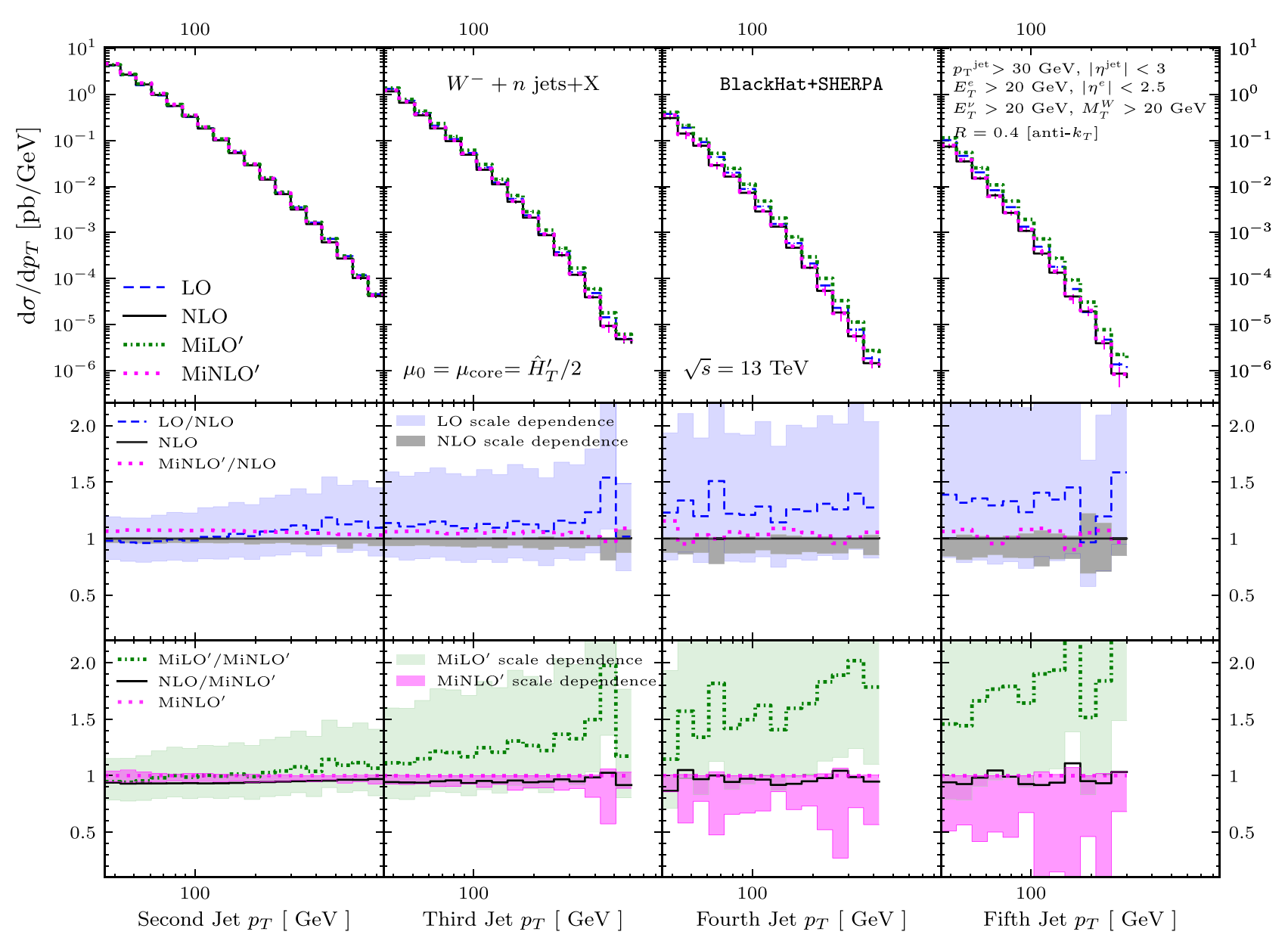

FIG. 5. $\quad p_{\mathrm{T}}$ distribution of the softest jet in inclusive samples of $W^{-}+n$ jets $(n=2,3,4,5)$ at the LHC at $\sqrt{s}=13$ TeV. In the upper panels, NLO predictions are shown as solid (black) lines and MiNLO' predictions as dotted (magenta) lines, while LO predictions are shown as dashed (blue) lines and MiLO' predictions as dash-dotted (green) lines. The central panels show the predictions for both the LO and MiNLO' distributions. We also show scale-dependence bands obtained by correlated variations by constant factors, as described in Sec. II C, normalized to the central NLO prediction in dark gray for the NLO results and in blue for the LO results. Similarly at the bottom, we show predictions for MiLO' and NLO distributions as well as scale-dependence bands for MiLO' in green and MiNLO' in magenta normalized to the central MiNLO' predictions.

for the different vector bosons $W^{ \pm}$and $Z$. Also, we only include $(\mathrm{N}) \mathrm{LO}$ and $\mathrm{Mi}(\mathrm{N}) \mathrm{LO}^{\prime}$ results, as the associated results with changing $\hat{H}_{\mathrm{T}}^{\prime} / 2$ to $\hat{S}_{\mathrm{T}}$ are rather consistent.

Figure 5 displays the $n$-th jet transverse momentum spectra in the calculation of $W^{-}+n$ jets for $n=2$ to 5 . The solid (black) lines show NLO predictions, the dotted (magenta) lines $\mathrm{MiNLO}^{\prime}$ predictions, while the dashed (blue) lines show LO predictions and the dash-dotted lines MiLO' predictions. The error bars represent the estimate of the statistical integration errors. The middle panels show ratios to the NLO result including scale dependence bands at LO and NLO. Similarly, the lower panels show ratios to the MiNLO' results and scale dependences for MiLO' and MiNLO'. Previous studies at lower energies (see, for example, [10]) have shown that the $n$-th jet $p_{\mathrm{T}}$ spectrum in an inclusive $V+n$ jets sample tends to have rather small distortions due to QCD corrections (as long as $n>1$ ). Our current study confirms this result, with the LO to NLO ratios being flat over a wide $p_{T}$ range. It is clear that although $\mathrm{LO}$ results employing an $\hat{H}_{\mathrm{T}}^{\prime} / 2$ dynamical scale have similar shapes to the NLO results, their normalization is very badly estimated, following the trends described in the previous section on total cross sections. We also notice that the MiNLO' results are in very good agreement with the NLO results in both shape and normalization over the seven orders of magnitude shown for the differential cross sections. This confirms that the predictions from NLO results for these observables are under good theoretical control, with uncertainties of order $15 \%$ (considering the $\hat{H}_{\mathrm{T}}^{\prime} / 2$ scale-dependence band as well as its deviation with respect to the MiNLO' result). We notice that for the fourth and fifth jet $p_{T}$ spectra the MiNLO' scale-dependence bands are considerably larger as compared to the fixed-order scale-dependence bands. Without considering fluctuations (attributable to statistical integration errors), these bands imply a scale uncertainty of up to $40 \%$. 


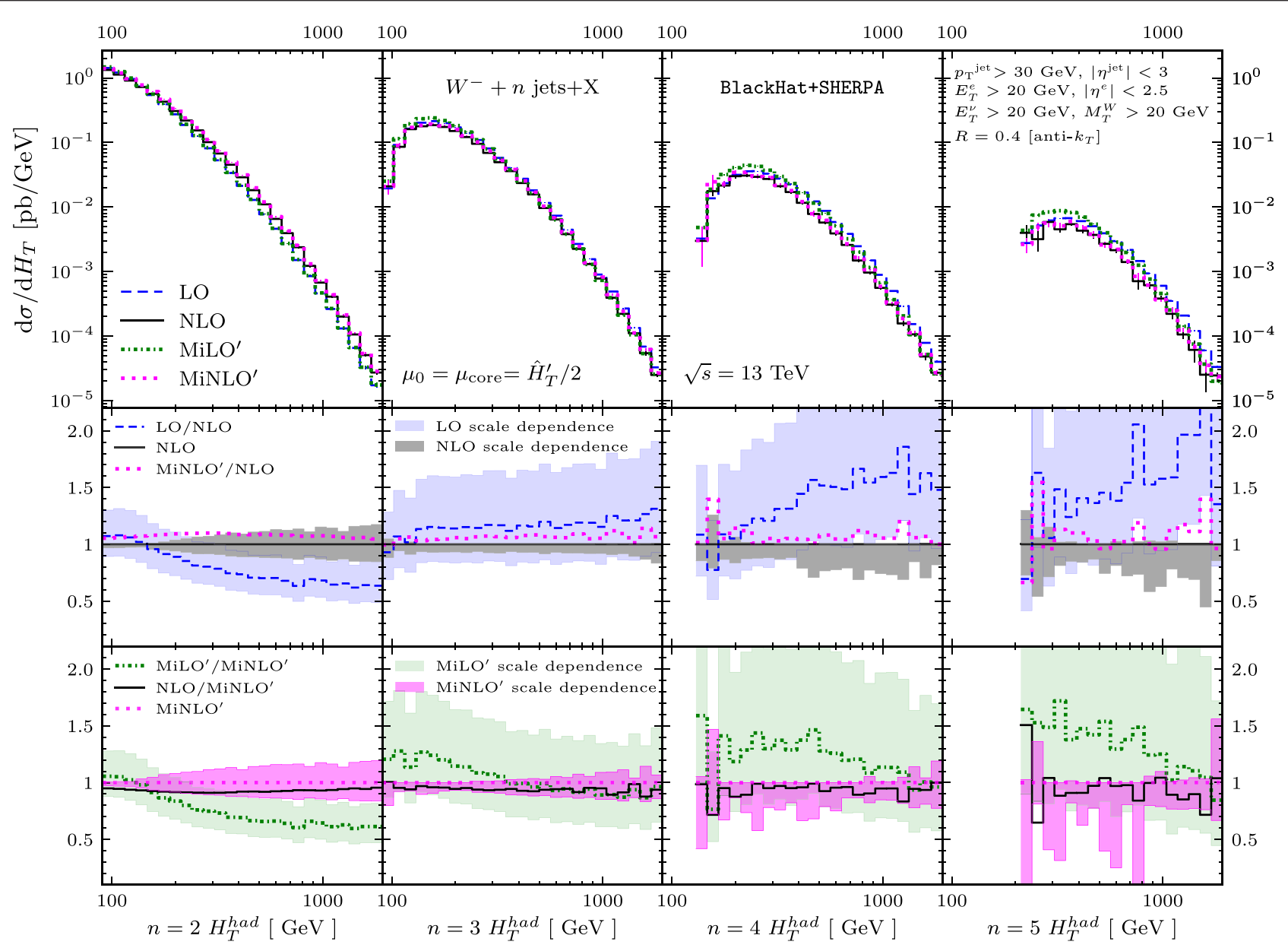

FIG. 6. Distribution in the total hadronic transverse energy $H_{T}$ in inclusive samples of $W^{-}+2,3,4,5$-jets. Format as in Fig. 5 .

We study the distribution in the total jet hadronic transverse energy $H_{\mathrm{T}}$ in Fig. 6 as well as ratios thereof between the MiNLO' and $\hat{H}_{\mathrm{T}}^{\prime} / 2$ scale choices in Fig. 7. The format of Fig. 6 is the same as that in Fig. 5. We show in side-by-side panels results for $W^{-}+n$ jets, with $n=2,3,4$, and 5. In general, we find that for $n \geq 3$, the corrections to the $H_{\mathrm{T}}$ spectrum change the shape of the distribution only mildly, which can be seen by looking at both the LO to NLO ratios as well as the MiNLO' to NLO ratios. Notice that the fluctuations at NLO for small values of $H_{\mathrm{T}}$, as we increase multiplicity, are just due to the fact that near threshold the integration errors grow large. On the other hand, the $n=2 \mathrm{LO}$ predictions have a large shape difference compared to that of the NLO results. These changes are similar to the corresponding observable for $n=1$ for which it is well known that large corrections appear from configurations with many jets in the final state [30]. The widening of the NLO scale band indeed shows that real contributions are large in the tail of the distribution, making this observable sensitive to quantum corrections (an associated observation for the same observable but at a hadron collider running at $100 \mathrm{TeV}$ was made in [50]). In principle, a computation of NNLO QCD correction to $V+2$ jets would be desirable in order to stabilize the predictions for this highly relevant process.

We notice a different behavior regarding the comparisons between $\mathrm{MiLO}^{\prime}$ and MiNLO' in the lower panels of Fig. 6 as well as in the MiLO' to LO ratios shown in Fig. 7. This arises from the fact that very large values of $H_{T}$ are mostly generated in events of dijet type with largely disparate scales of jet production, i.e., $p_{T, j 1} \approx p_{T, j 2} \gg p_{T, j 3}, \ldots, p_{T, j n}$. This induces Sudakov suppression factors that reduce the corresponding high$H_{T}$ tails and improve the agreement with the NLO prediction. At NLO, the ratios in Fig. 7 are quite stable and around 1.

Figure 8 shows that the differential cross section of the transverse momentum of the $W^{-}$boson is well behaved under quantum corrections. Indeed, these steeply falling distributions show very similar shapes for LO, NLO, MiLO', and MiNLO' results. This distribution is interesting from the experimental point of view, not only for its relation to several searches for beyond the SM physics but also of its association to having under control known missing energy signatures. 


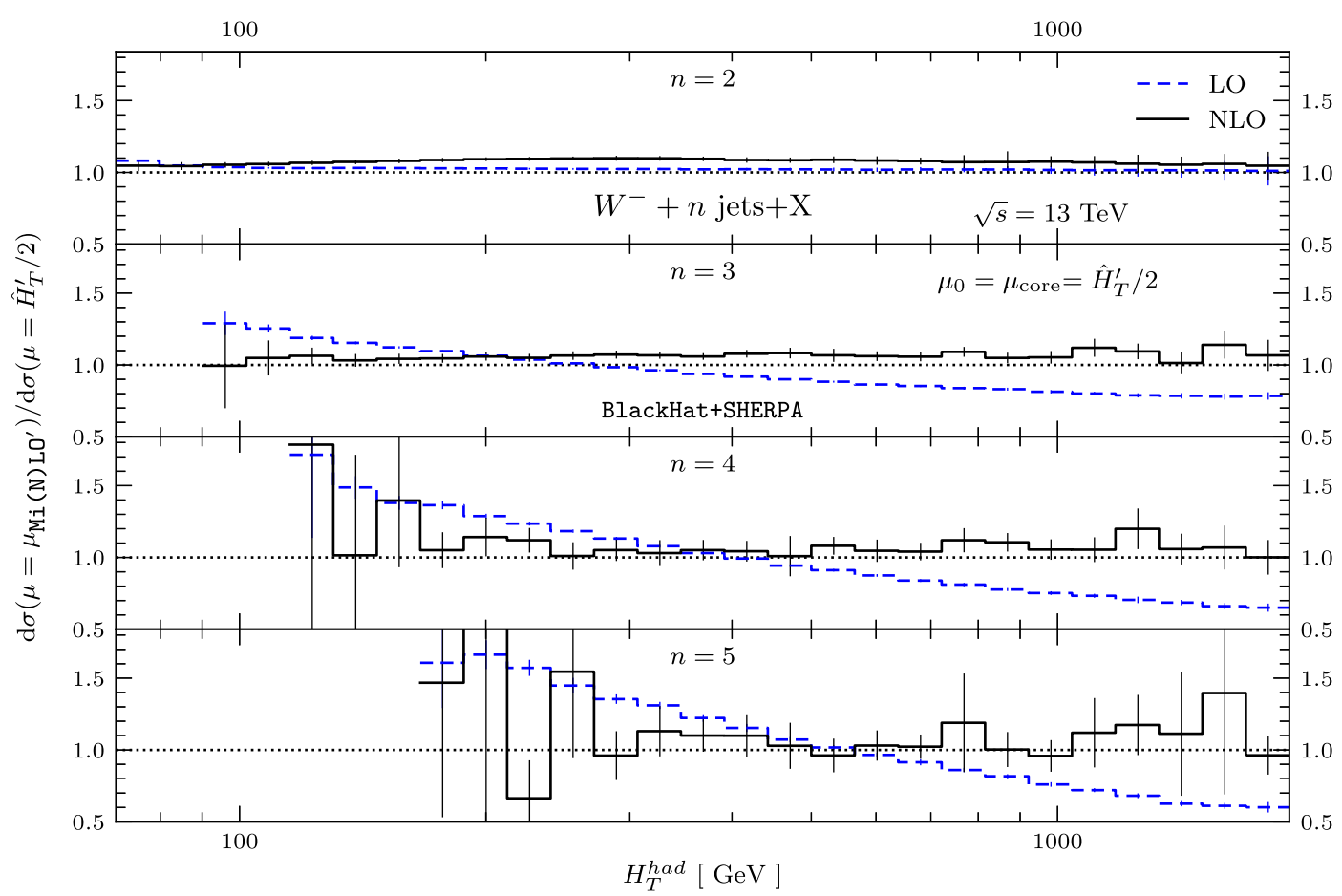

FIG. 7. $\mathrm{Mi}(\mathrm{N}) \mathrm{LO}^{\prime}$ to $\hat{H}_{\mathrm{T}}^{\prime} / 2$ ratio at both $\mathrm{LO}$ and NLO for the distribution in the total hadronic transverse energy $H_{T}$ in inclusive samples of $W^{-}+n$-jet production $(n=2,3,4$ and 5).

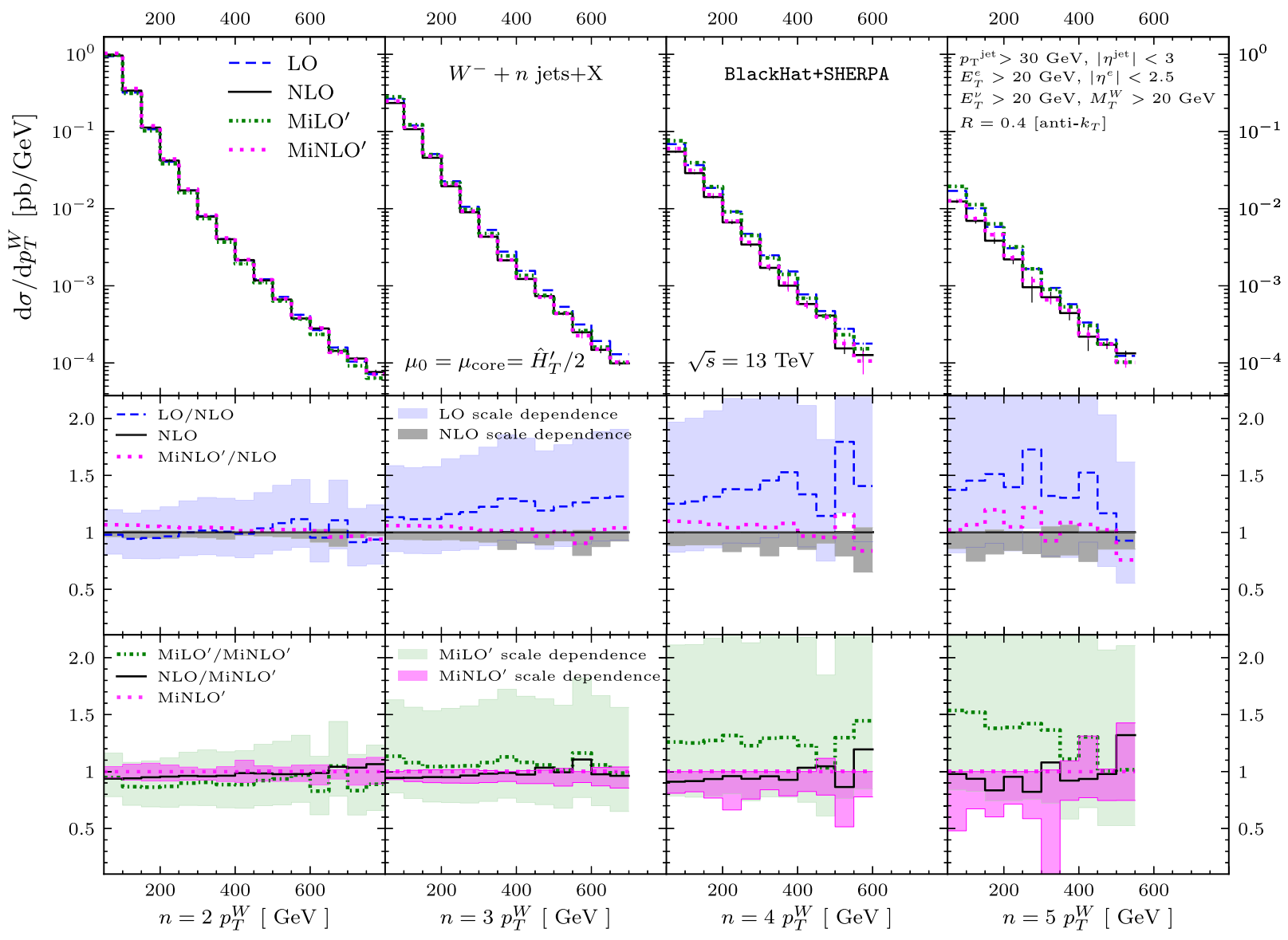

FIG. 8. Distribution in the transverse energy of the $W^{-}$boson in inclusive samples of $W^{-}+2,3,4,5$-jets. Format as in Fig. 5 . 


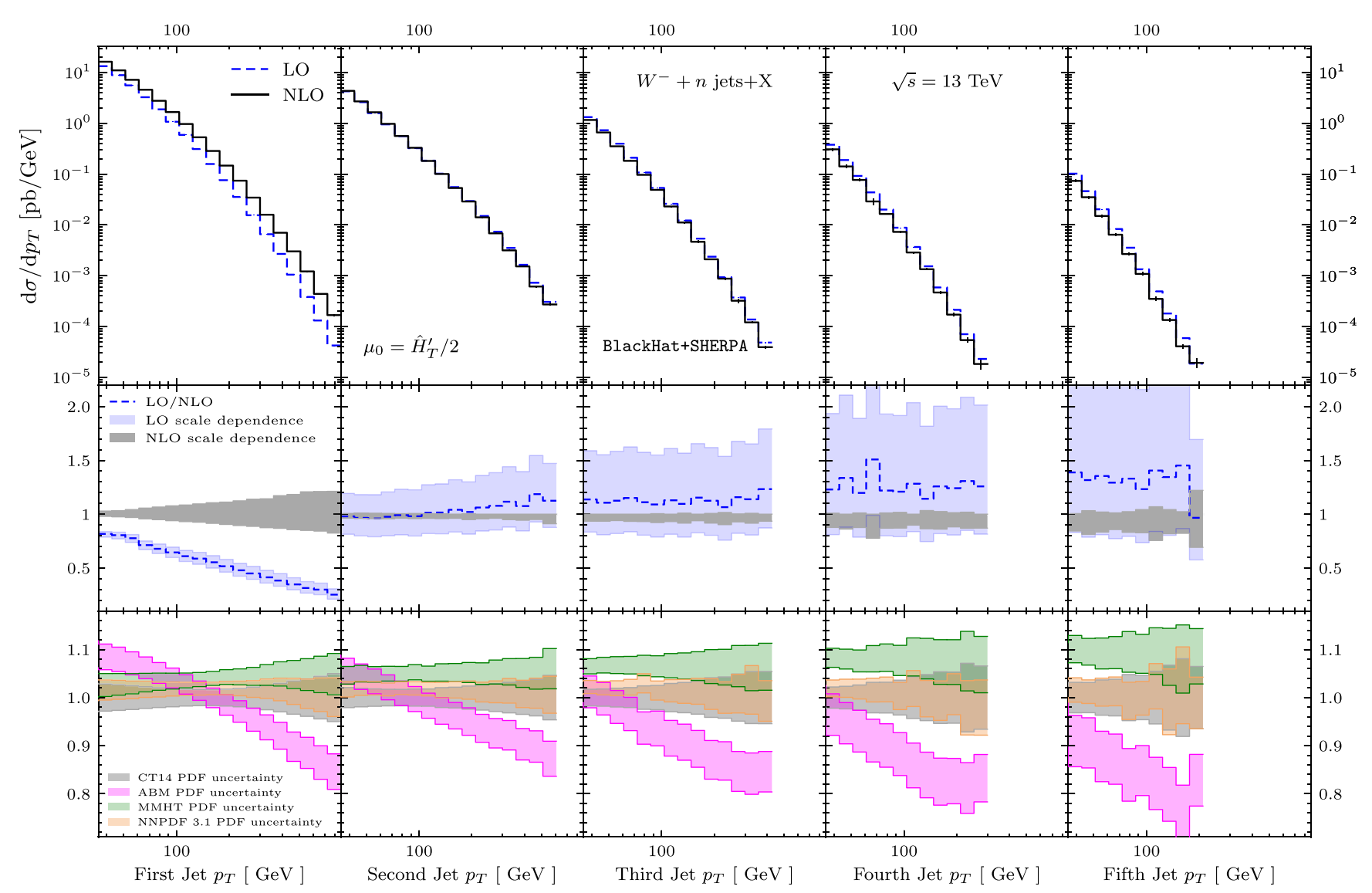

FIG. 9. PDF uncertainty for the $p_{\mathrm{T}}$ distribution of the softest jet in an inclusive sample of $W^{-}+n$ jets $(n=1,2,3,4,5)$ at the LHC at $\sqrt{s}=13 \mathrm{TeV}$. In the upper panels, the NLO predictions are shown as solid (black) lines, while the LO predictions are shown as dashed (blue) lines. The central panels show the scale-dependence bands normalized to the central NLO prediction in dark gray for NLO and blue for LO. The lower panels show the NLO PDF uncertainty for the CT14 (gray), ABM (magenta), MMHT (green), and NNPDF 3.1 (orange) PDF sets, normalized to the results obtained with CT14.

In Fig. 9, we explore uncertainties associated with the PDFs and show the PDF uncertainty bands for $n$ th-jet $p_{\mathrm{T}}$ spectra in an inclusive sample of $W^{-}+$jets for $\hat{H}_{\mathrm{T}}^{\prime} / 2$, analogous to the distributions shown in Fig. 5. The additional bottom panel shows the NLO PDF uncertainty bands for the CT14 (gray) [33], ABM (magenta) [34], MMHT (green) [35], and NNPDF 3.1 (orange) [36] PDF sets, and we normalize to the central value obtained with CT14. The data for Fig. 9 was generated by creating a fastNLO table [51] from the $n$-tuple data. We also show the central values for total cross sections obtained with the different

TABLE V. PDF variations of the NLO QCD total cross sections for inclusive $W^{-}+1,2,3,4,5$-jet production normalized to the results for CT14. These data were generated by creating a fastNLO table [51] from the $n$-tuple data.

\begin{tabular}{lccccc}
\hline \hline nbr. of jets & 1 & 2 & 3 & 4 & 5 \\
\hline CT14 & 1.000 & 1.000 & 1.000 & 1.000 & 1.000 \\
ABM & 1.070 & 1.039 & 1.000 & 0.960 & 0.920 \\
MMHT & 1.029 & 1.049 & 1.066 & 1.080 & 1.095 \\
NNPDF 3.1 & 1.016 & 1.018 & 1.020 & 1.020 & 1.016 \\
\hline \hline
\end{tabular}

choices of PDF sets, normalized to the results for CT14, in Table V.

We observe that PDF uncertainties can reach values of up to $10 \%$ and that most of the error sets overlap. Nevertheless, both central value and uncertainty bands of the ABM results lay outside the uncertainty bands of all other PDF sets. Also, the MMHT bands lay systematically higher than the others, a trend that is more pronounced in the large-multiplicity cases. It was observed previously that the inclusion of NNLO corrections in inclusive $\mathrm{Z}+$ jet production leads to a better agreement between MMHT results and those obtained with CT14 and NNPDF [52].

All uncertainty bands increase at larger $p_{\mathrm{T}}$, as the effective mass sampled for the corresponding events grows, and the PDFs are evaluated for larger values of the Bjorken $x$, with less data available to constrain the PDF fits. PDF uncertainties are thus of the same order as NLO scale uncertainties, in particular for the high-multiplicity processes, where we observe a considerable spread between the different PDF sets. At the level of normalized NLO QCD total cross sections, this is shown in Table V. 

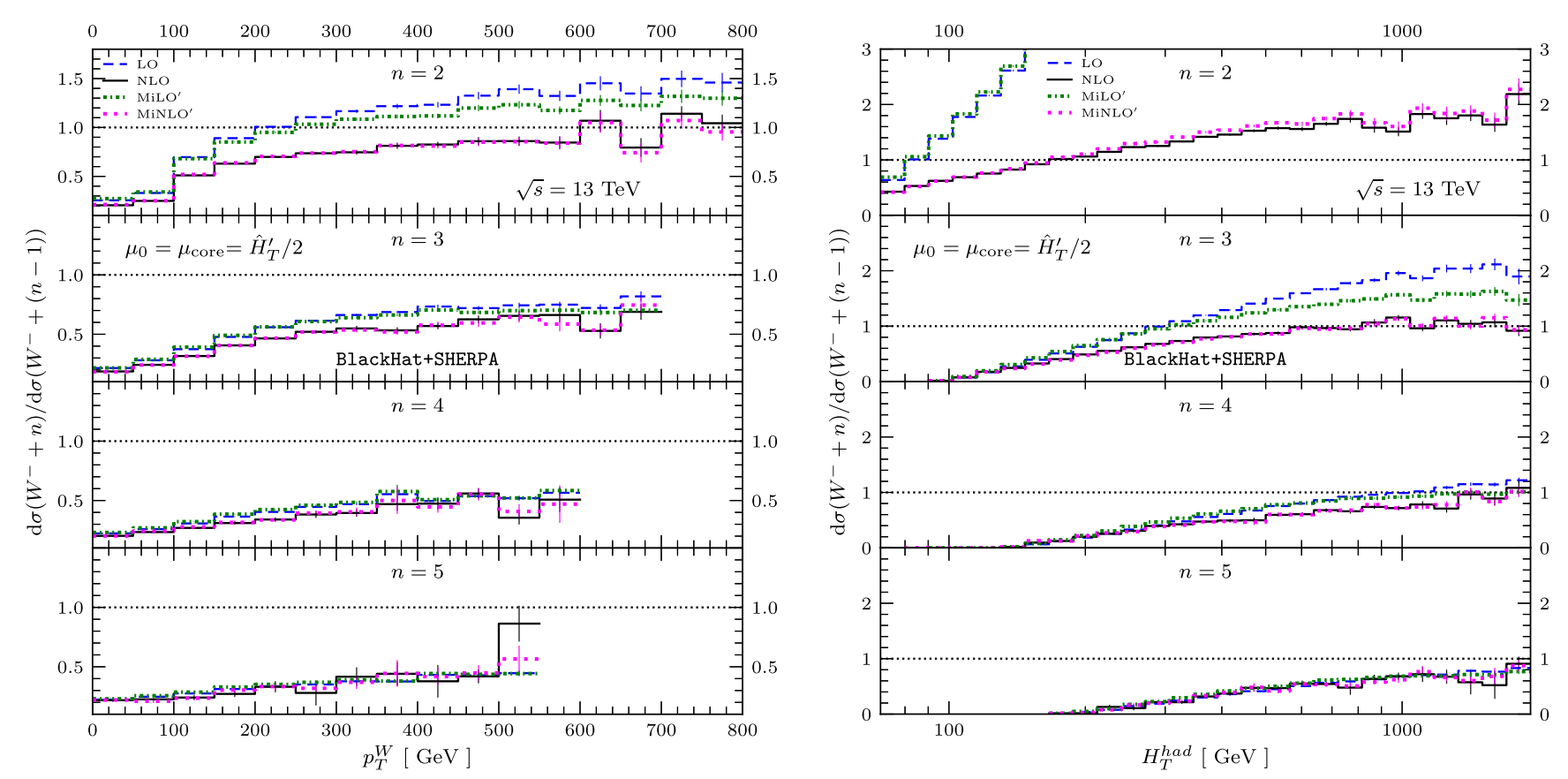

FIG. 10. Ratios of $W^{-}+n$-jet to $W^{-}+(n-1)$-jet cross sections as a function of the $W^{-}$transverse momentum $p_{\mathrm{T}}^{W}$ on the left and as a function of the hadronic transverse energy $H_{\mathrm{T}}$ on the right. LO results are shown as dashed (blue) lines and NLO results as solid (black) lines, while MiLO' results are shown as dash-dotted (green) lines and MiNLO' results as dotted (magenta) lines. We present results from $n=2$ (top panel) to $n=5$ (bottom panel).

\section{CROSS SECTION RATIOS}

In this section, we study a series of observable ratios, which feature reduced uncertainties compared to basic observables such as cross sections or differential distributions. In particular, experimental uncertainties related to jet energy scale, lepton efficiency, acceptance, and protonproton luminosity should be greatly reduced. The observable ratios are also expected to suffer less from theoretical uncertainties from uncalculated higher-order corrections. Consequently, these ratios are helpful to better understand the structure of quantum corrections to processes with a vector boson in association with multiple jets. As shown in [22], they exhibit certain universal features that can be exploited in phenomenological studies at hadron colliders. In this section, we study the differential jet ratio for $W^{-}$ production as well as differential flavor ratios of both $W^{-} / W^{+}$and $Z / W$ production.

\section{A. Jet ratios}

We first examine the dependence of the jet-production ratio on the $W^{-}$-boson transverse momentum $p_{\mathrm{T}}^{W}$ and total hadronic transverse energy $H_{\mathrm{T}}$. We display the differential cross section ratios for the associated production with up to 5 jets in Fig. 10, where the $p_{\mathrm{T}}^{W}$ distributions are shown in the left panel and the $H_{\mathrm{T}}$ distribution in the right panel. The ratios are shown for both scale choices with those for $\hat{H}_{\mathrm{T}}^{\prime} / 2$ shown at $\mathrm{LO}$ as dashed (blue) lines and at NLO as solid (black) lines, while MiLO' ratios are shown as dash-dotted (green) lines and those for MiNLO' as dotted (magenta) lines.

As a function of $p_{\mathrm{T}}^{W}$, we observe that the $W^{-}+$ 2 -jet $/ W^{-}+1$-jet ratio $(n=2)$ shows large NLO corrections. This is mainly due to the large corrections appearing at NLO [30], which are stabilized at NNLO [7]. In the lowest $p_{\mathrm{T}}$ region (up to the order of the $W$ mass), the ratios lie around a value of 0.25 , roughly independent of the number of jets. The NLO corrections are modest for all displayed multiplicities in this region, being in agreement with the total cross sections displayed in Table II. With higher $p_{\mathrm{T}}^{W}$, the ratios grow monotonically, and for $n \geq 3$, they stabilize for large values of $p_{\mathrm{T}}^{W}$. For more jets, the increase is less pronounced, and the ratio stabilizes for $n=5$ around a value 0.5 . For the low-multiplicity cases $(n \leq 3)$, and in particular for large values of $p_{\mathrm{T}}^{W}$, the ratio for MiLO' lies below that for LO. The ratios for higher multiplicities as well as all those of NLO and MiNLO' agree very well.

Similar to the $p_{\mathrm{T}}^{W}$ ratios, we observe that the differential ratio in $H_{\mathrm{T}}$ for $n=2$ is not stable with the fixed-order quantum corrections. Even at NLO, the differential jet production ratio is larger than 1 for a large part of $H_{\mathrm{T}}$ shown. We would expect this observable to stabilize as higher-multiplicity results are included either through NNLO or higher-order calculations, or through multijet merging at NLO. Around the threshold, all ratios lie at a value of the same order. With increasing $H_{\mathrm{T}}$, they again increase monotonically and at NLO stabilize for large values of $H_{\mathrm{T}}$. Looking at the high $H_{\mathrm{T}}$ region, the ratios 

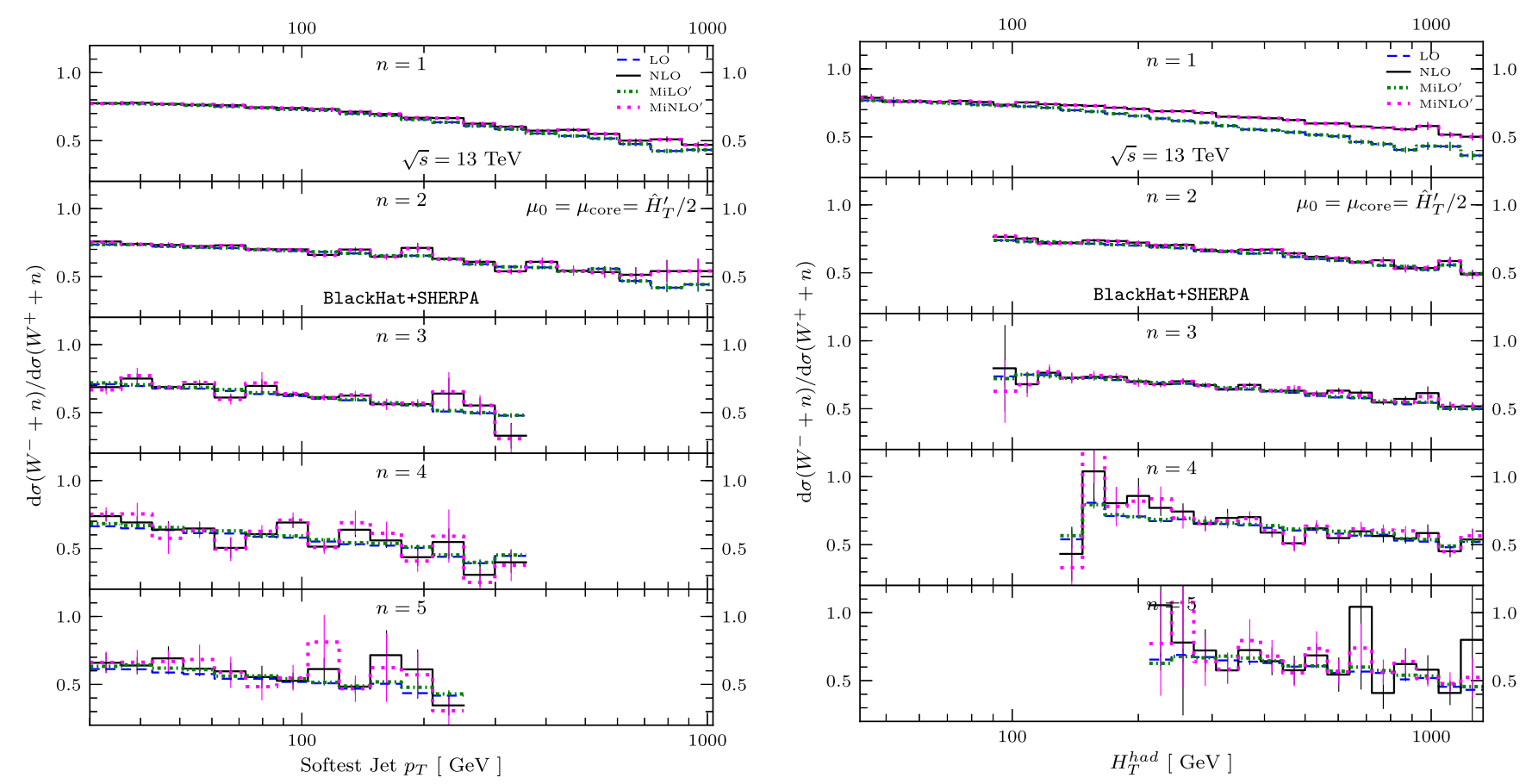

FIG. 11. Charge ratios of $W^{-}+n$-jet to $W^{+}+n$-jet cross sections as a function of the softest-jet $p_{\mathrm{T}}$ on the left and as a function of the hadronic transverse energy $H_{\mathrm{T}}$ on the right for results from $n=2$ (top panel) to $n=5$ (bottom panel). Format as in Fig. 10 .

show a characteristic behavior. Such events tend to be populated by multiple jets, and the $H_{\mathrm{T}}$ distributions in these jet bins tend to overlap [22], which results in the ratio tending to 1. As for the previous distributions, MiNLO' and NLO ratios agree very well, with a slight discrepancy between LO and MiLO' ratios for the lower multiplicity cases.

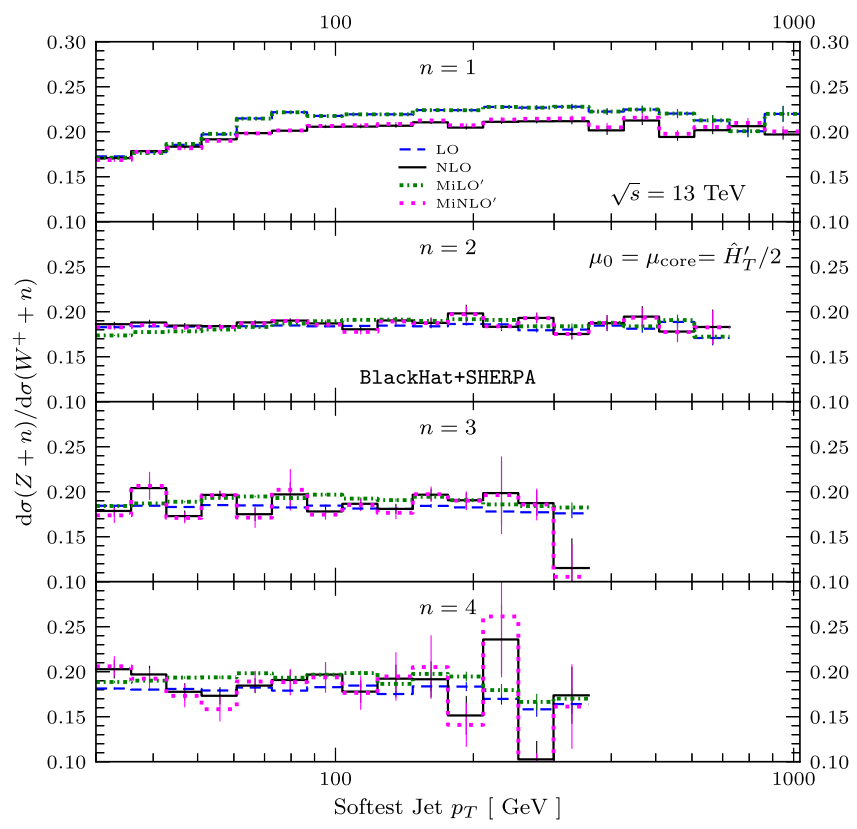

\section{B. Flavor ratios}

In this subsection, we show flavor ratios of $W^{-}$to $W^{+}$ production as well as $Z$ to $W^{+}$production. These ratios are of particular benefit as they are reliable observables for the extraction of valence-quark PDF information for large values of Bjorken $x[22,53]$.

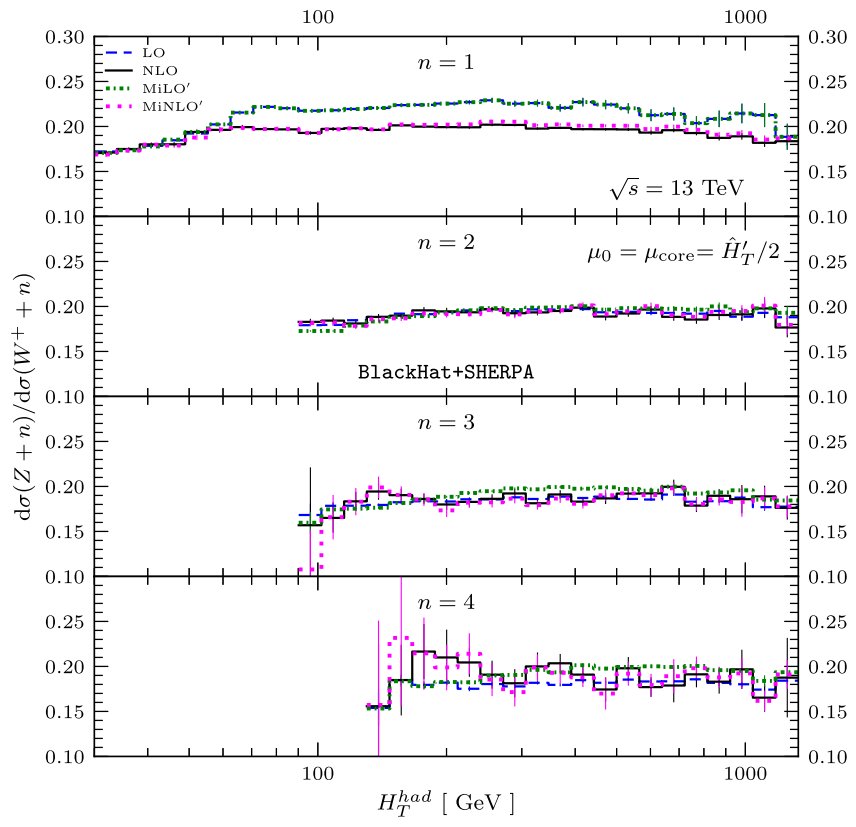

FIG. 12. Ratios of $Z+n$-jet to $W^{+}+n$-jet cross sections as a function of the softest-jet $p_{\mathrm{T}}$ on the left and as a function of the hadronic transverse energy $H_{\mathrm{T}}$ on the right. We present results from $n=1$ (top panel) to $n=4$ (bottom panel). Format as in Fig. 10 . 
In Figs. 11 and 12, we display differential ratios as a function of both the transverse momentum $p_{\mathrm{T}}$ of the softest jet (left panel) and of the total hadronic energy $H_{\mathrm{T}}$ (right panel) for $W^{-}$to $W^{+}$accompanied with up to five jets and $Z$ to $W^{+}$with up to four jets, respectively. The ratios are shown for both scale choices with those for $\hat{H}_{\mathrm{T}}^{\prime} / 2$ shown at LO as dashed (blue) lines and at NLO as solid (black) lines, while MiLO' ratios are shown as dash-dotted (green) lines and those for MiNLO' as dotted (magenta) lines. Experimental studies on the latter ratios have been made, for example, in [4].

The $W^{-} / W^{+}$ratios as a function of the transverse momentum for low values of $p_{\mathrm{T}}$ lie around a value of roughly the same order, at about 0.8 for $n=2$, for all shown multiplicities $n$. With increasing $p_{\mathrm{T}}$, we see a monotonic decrease of the corresponding ratio. We attribute this decrease to the dominance of $u$ quarks over $d$ quarks at large values of Bjorken $x$. The NLO corrections to the transverse momentum ratios are mild, and the results for both scale choices agree very well.

We observe a similar behavior for the differential ratios as a function of the total hadronic energy $H_{\mathrm{T}}$. In particular, the ratios decrease monotonically with increasing $H_{\mathrm{T}}$ and take values of the same order at low $H_{\mathrm{T}}$ for all multiplicities; also, the results for both scale choices agree very well. However, we observe noticable NLO correction for the $n=1$ case in the high $H_{\mathrm{T}}$ tail.

The ratios of $Z$ to $W^{+}$production in Fig. 12 are quite flat over the full range of variables shown, which is in contrast to the associated charge-asymmetry ratios in Fig. 11. The values of $Z / W^{+}$ratios as a function of both variables shown lie around 0.2 for all multiplicities $n$ shown. This constant value shows the dominance of $u$-quark distribution for both $Z$ and $W^{+}$production when the sampled parton fraction $x$ is large. A small decrease of the $Z / W^{+}$ratio can be observed in the low $p_{\mathrm{T}}$ and low $H_{T}$ regions in the ratio for $n=1$. Also for all the $Z / W^{+}$ratios, the results for both scale choices agree very well. Our results show that the quantum corrections in the $Z / W^{+}$observable ratios are quite mild, which make them excellent choices for searches for new physics.

\section{CONCLUSIONS}

In this paper, we presented NLO QCD predictions for the production of an electroweak gauge boson with up to five jets for $W^{ \pm}$and with up to four jets for $Z$, in the final state for the LHC with $\sqrt{s}=13 \mathrm{TeV}$, extending previous predictions $[10,11]$ to the energy of LHC Run II. Since these processes constitute an important background to many new physics searches involving missing energy, as well as precise top-quark measurements, the theoretical predictions we provide are an important ingredient to fully exploit the discovery potential of the LHC in the upcoming years.
We observed that the scale dependence in NLO predictions for both total cross sections and differential distributions is strongly reduced compared to those at LO. Similar to the $\sqrt{s}=7 \mathrm{TeV}$ case, the scale dependence of total cross sections shrinks from up to $50 \%$ at $\mathrm{LO}$ to a sensitivity of about $15 \%$ at NLO. We also compared results obtained with several other functional forms of dynamical scales, most notably comparing results with fixed-order scales like $\hat{H}_{\mathrm{T}}^{\prime} / 2$ with the MiNLO' (original formulation in [31]) reweighting procedure. This is the first time this comparison is carried out in the context of processes with high jet multiplicity. For total cross sections, as well as for differential distributions, NLO results obtained with $\hat{H}_{\mathrm{T}}^{\prime} / 2$ and $\mathrm{MiNLO}^{\prime}$ are largely consistent in both shape and normalization. There is also good agreement in the observed scale sensitivity with both types of scales, although for the specific case of the softest jet $p_{T}$ distribution in high-multiplicity processes we find a considerably larger MiNLO' scale-dependence band (up to a factor of 2.7 larger with respect to the fixed-order result). More dedicated studies of the enhanced scale sensitivity with the MiNLO' scheme is left to future work. We highlight the general good agreement for observables associated to high-multiplicity processes that NLO QCD predictions with $\hat{H}_{\mathrm{T}}^{\prime} / 2$ and $\mathrm{MiNLO}$ ' scales show, giving confidence that those represent first numerically reliable predictions.

We furthermore computed several observable ratios and found that jet-production ratios have an increased stability compared to the results at $\sqrt{s}=7 \mathrm{TeV}$. We thereby have found a setup for which there is independence of the jetproduction ratios on the number of jets.

The present study provides insight into the phenomenologically relevant process class of electroweak gauge boson $\left(W^{ \pm}, Z\right)$ production plus up to five jets at $\sqrt{s}=13 \mathrm{TeV}$. We look forward to more comparisons of our results, as the ones recently shown in [5], with LHC data at the energy frontier.

\section{ACKNOWLEDGMENTS}

We thank Z. Bern, L. J. Dixon, H. Ita, and D. A. Kosower for many discussions. We also thank Keith Hamilton for help with the detailed cross-check of our MiNLO implementation and for advice regarding the systematic uncertainties. The work of F. R. A. and F. F. C. is supported by the Alexander von Humboldt Foundation, in the framework of the Sofja Kovalevskaja Award 2014, endowed by the German Federal Ministry of Education and Research. S. H.'s work was supported by the U.S. Department of Energy under Contract No. DE-AC02-76SF00515. This work was performed on the bwUniCluster funded by the Ministry of Science, Research and the Arts BadenWürttemberg and the Universities of the State of 
Baden-Württemberg, Germany, within the framework program bwHP. Parts of the calculation have been completed at the National Energy Research Scientific Computing
Center, a DOE Office of Science User Facility supported by the Office of Science of the U.S. Department of Energy under Contract No. DE-AC02-05CH11231.
[1] G. Aad et al. (ATLAS Collaboration), Measurements of the $\mathrm{W}$ production cross sections in association with jets with the ATLAS detector, Eur. Phys. J. C 75, 82 (2015); A measurement of the ratio of the production cross sections for $W$ and $Z$ bosons in association with jets with the ATLAS detector, Eur. Phys. J. C 74, 3168 (2014); Measurement of the production cross section of jets in association with a $\mathrm{Z}$ boson in pp collisions at $\sqrt{s}=7 \mathrm{TeV}$ with the ATLAS detector, J. High Energy Phys. 07 (2013) 032; Study of jets produced in association with a $W$ boson in $p p$ collisions at $\sqrt{s}=7 \mathrm{TeV}$ with the ATLAS detector, Phys. Rev. D 85, 092002 (2012); Measurement of the production cross section for $Z / \gamma *$ in association with jets in $p p$ collisions at $\sqrt{s}=7 \mathrm{TeV}$ with the ATLAS detector, Phys. Rev. D 85 (2012) 032009; A measurement of the ratio of the $W$ and $Z$ cross sections with exactly one associated jet in $p p$ collisions at $\sqrt{s}=7 \mathrm{TeV}$ with ATLAS, Phys. Lett. B 708, 221 (2012); Measurement of the production cross section for W-bosons in association with jets in $p p$ collisions at $\sqrt{s}=7 \mathrm{TeV}$ with the ATLAS detector, Phys. Lett. B 698, 325 (2011).

[2] V. Khachatryan et al. (CMS Collaboration), Comparison of the $Z / \gamma+$ jets to $\gamma+$ jets cross sections in $p p$ collisions at $\sqrt{s}=8 \mathrm{TeV}$, J. High Energy Phys. 10 (2015) 128; Erratum, J. High Energy Phys. 04 (2016) 010(E); Measurements of differential cross sections for associated production of a W boson and jets in proton-proton collisions at $\sqrt{s}=8 \mathrm{TeV}$, Phys. Rev. D 95, 052002 (2017); Measurements of the differential production cross sections for a $\mathrm{Z}$ boson in association with jets in pp collisions at $\sqrt{s}=8 \mathrm{TeV}$, J. High Energy Phys. 04 (2017) 022.

[3] V. Khachatryan et al. (CMS Collaboration), Measurements of jet multiplicity and differential production cross sections of $Z+$ jets events in proton-proton collisions at $\sqrt{s}=7$ TeV, Phys. Rev. D 91, 052008 (2015); Differential cross section measurements for the production of a W boson in association with jets in proton-proton collisions at $\sqrt{s}=7 \mathrm{TeV}$, Phys. Lett. B 741, 12 (2015); Event shapes and azimuthal correlations in $Z+$ jets events in $p p$ collisions at $\sqrt{s}=7 \mathrm{TeV}$, Phys. Lett. B 722, 238 (2013); Measurement of the Polarization of W Bosons with Large Transverse Momenta in W + Jets Events at the LHC, Phys. Rev. Lett. 107, 021802 (2011).

[4] S. Chatrchyan et al. (CMS Collaboration), Jet production rates in association with $\mathrm{W}$ and $\mathrm{Z}$ bosons in pp collisions at sqrt(s) = 7 TeV, J. High Energy Phys. 01 (2012) 010.

[5] M. Aaboud et al. (ATLAS Collaboration), Measurements of the production cross section of a $Z$ boson in association with jets in pp collisions at $\sqrt{s}=13 \mathrm{TeV}$ with the ATLAS detector, Eur. Phys. J. C 77, 361 (2017).
[6] A. M. Sirunyan et al. (CMS Collaboration), Measurement of the differential cross sections for the associated production of a $W$ boson and jets in proton-proton collisions at $\sqrt{s}=13$ TeV, Phys. Rev. D 96, 072005 (2017).

[7] R. Boughezal, C. Focke, X. Liu, and F. Petriello, W-boson Production in Association with a Jet at Next-to-Next-toLeading Order in Perturbative QCD, Phys. Rev. Lett. 115, 062002 (2015); R. Boughezal, J. M. Campbell, R. K. Ellis, C. Focke, W. T. Giele, X. Liu, and F. Petriello, Z-boson Production in Association with a Jet at Next-to-Next-toLeading Order in Perturbative QCD, Phys. Rev. Lett. 116, 152001 (2016); A. Gehrmann-De Ridder, T. Gehrmann, E. W. N. Glover, A. Huss, and T. A. Morgan, Z+jet production at NNLO, arXiv:1607.01749.

[8] J. M. Lindert et al., Precise predictions for V+jets dark matter backgrounds, Eur. Phys. J. C 77, 829 (2017).

[9] C. F. Berger, Z. Bern, L. J. Dixon, F. Febres Cordero, D. Forde, T. Gleisberg, H. Ita, D. A. Kosower, and D. Maître, Precise Predictions for $W+4$ Jet Production at the Large Hadron Collider, Phys. Rev. Lett. 106, 092001 (2011).

[10] Z. Bern, L. J. Dixon, F. Febres Cordero, S. Höche, H. Ita, D. A. Kosower, D. Maître, and K. J. Ozeren, Next-to-leading order $\mathrm{W}+5$-jet production at the LHC, Phys. Rev. D 88, 014025 (2013).

[11] H. Ita, Z. Bern, L. J. Dixon, F. Febres Cordero, D. A. Kosower, and D. Maître, Precise predictions for $Z+4$ jets at Hadron Colliders, Phys. Rev. D 85, 031501 (2012).

[12] S. Höche, F. Krauss, M. Schönherr, and F. Siegert, $W+n$-Jet Predictions at the Large Hadron Collider at Next-to-Leading Order Matched with a Parton Shower, Phys. Rev. Lett. 110, 052001 (2013).

[13] S. Höche, F. Krauss, M. Schönherr, and F. Siegert, QCD matrix elements + parton showers: The NLO case, J. High Energy Phys. 04 (2013) 027; L. Lönnblad and S. Prestel, Merging multi-leg NLO matrix elements with parton showers, J. High Energy Phys. 03 (2013) 166; R. Frederix and S. Frixione, Merging meets matching in MC@NLO, J. High Energy Phys. 12 (2012) 061.

[14] A. Denner, L. Hofer, A. Scharf, and S. Uccirati, Electroweak corrections to lepton pair production in association with two hard jets at the LHC, J. High Energy Phys. 01 (2015) 094; S. Kallweit, J. M. Lindert, P. Maierhöfer, S. Pozzorini, and M. Schönherr, NLO electroweak automation and precise predictions for $\mathrm{W}+$ multijet production at the LHC, J. High Energy Phys. 04 (2015) 012.

[15] S. Kallweit, J. M. Lindert, P. Maierhöfer, S. Pozzorini, and M. Schönherr, NLO QCD + EW predictions for $\mathrm{V}+$ jets including off-shell vector-boson decays and multijet merging, J. High Energy Phys. 04 (2016) 021. 
[16] C. F. Berger, Z. Bern, L. J. Dixon, F. Febres Cordero, D. Forde, H. Ita, D. A. Kosower, and D. Maître, An automated implementation of on-shell methods for one-loop amplitudes, Phys. Rev. D 78, 036003 (2008).

[17] R. Britto, Loop amplitudes in gauge theories: Modern analytic approaches, J. Phys. A 44, 454006 (2011); R. K. Ellis, Z. Kunszt, K. Melnikov, and G. Zanderighi, One-loop calculations in quantum field theory: from Feynman diagrams to unitarity cuts, Phys. Rep. 518, 141 (2012); H. Ita, Susy theories and QCD: Numerical approaches, J. Phys. A 44, 454005 (2011).

[18] C. F. Berger, Z. Bern, L. J. Dixon, F. Febres Cordero, D. Forde, T. Gleisberg, H. Ita, D. A. Kosower, and D. Maître, Precise Predictions for $W+3$ Jet Production at Hadron Colliders, Phys. Rev. Lett. 102, 222001 (2009).

[19] C.F. Berger, Z. Bern, L.J. Dixon, F. Febres Cordero, D. Forde, T. Gleisberg, H. Ita, D. A. Kosower, and D. Maître, Next-to-leading order QCD predictions for W+3-jet distributions at hadron colliders, Phys. Rev. D 80, 074036 (2009).

[20] C. F. Berger, Z. Bern, L. J. Dixon, F. Febres Cordero, D. Forde, T. Gleisberg, H. Ita, D. A. Kosower, and D. Maître, Next-toleading order QCD predictions for $Z, \gamma^{*}+3$-jet distributions at the Tevatron, Phys. Rev. D 82, 074002 (2010).

[21] Z. Bern, G. Diana, L. J. Dixon, F. Febres Cordero, S. Höche, D. A. Kosower, H. Ita, D. Maître, and K. Ozeren, Four-Jet Production at the Large Hadron Collider at Next-to-Leading Order in QCD, Phys. Rev. Lett. 109, 042001 (2012); Z. Bern, G. Diana, L. J. Dixon, F. Febres Cordero, S. Höche, H. Ita, D. A. Kosower, D. Maître, and K. J. Ozeren, Driving missing data at next-to-leading order, Phys. Rev. D 84, 114002 (2011); Missing energy and jets for supersymmetry searches, Phys. Rev. D 87, 034026 (2013); Z. Bern, L. J. Dixon, F. Febres Cordero, S. Höche, H. Ita, D. A. Kosower, N. A. Lo Presti, and D. Maître, Next-to-leading order gamma gamma + 2-jet production at the LHC, Phys. Rev. D 90, 054004 (2014); F. Febres Cordero, P. Hofmann, and $\mathrm{H}$. Ita, $W^{+} W^{-}+3$-jet production at the Large Hadron Collider in next-to-leading-order QCD, Phys. Rev. D 95, 034006 (2017).

[22] Z. Bern, L. J. Dixon, F. Febres Cordero, S. Höche, H. Ita, D. A. Kosower, and D. Maître, Extrapolating W-associated jetproduction ratios at the LHC, Phys. Rev. D 92, 014008 (2015).

[23] F. R. Anger, F. Febres Cordero, H. Ita, and V. Sotnikov, NLO QCD predictions for $W b \bar{b}$ production in association with up to three light jets at the LHC, Phys. Rev. D 97, 036018 (2018).

[24] T. Gleisberg, S. Höche, F. Krauss, A. Schälicke, S. Schumann, and J.C. Winter, SHERPA 1. alpha: A proof of concept version, J. High Energy Phys. 02 (2004) 056; T. Gleisberg, S. Höche, F. Krauss, M. Schönherr, S. Schumann, F. Siegert, and J. Winter, Event generation with SHERPA 1.1, J. High Energy Phys. 02 (2009) 007.

[25] S. Catani and M. H. Seymour, A general algorithm for calculating jet cross sections in NLO QCD, Nucl. Phys. B485, 291 (1997); Erratum, B510, 503 (1998).

[26] T. Gleisberg and S. Höche, Comix, a new matrix element generator, J. High Energy Phys. 12 (2008) 039.

[27] R. Brun and F. Rademakers, ROOT: An object oriented data analysis framework, Nucl. Instrum. Methods Phys. Res., Sect. A 389, 81 (1997).
[28] Z. Bern, L. J. Dixon, F. Febres Cordero, S. Höche, H. Ita, D. A. Kosower, and D. Maître, Ntuples for NLO events at Hadron Colliders, Comput. Phys. Commun. 185, 1443 (2014).

[29] N. Greiner, S. Höche, G. Luisoni, M. Schönherr, and J. C. Winter, Full mass dependence in Higgs boson production in association with jets at the LHC and FCC, J. High Energy Phys. 01 (2017) 091.

[30] M. Rubin, G. P. Salam, and S. Sapeta, Giant QCD K-factors beyond NLO, J. High Energy Phys. 09 (2010) 084.

[31] K. Hamilton, P. Nason, and G. Zanderighi, MINLO: Multiscale improved NLO, J. High Energy Phys. 10 (2012) 155.

[32] S. Höche, P. Maierhoefer, N. Moretti, S. Pozzorini, and F. Siegert, Next-to-leading order QCD predictions for topquark pair production with up to three jets, Eur. Phys. J. C 77, 145 (2017).

[33] S. Dulat, T.-J. Hou, J. Gao, M. Guzzi, J. Huston, P. Nadolsky, J. Pumplin, C. Schmidt, D. Stump, and C.-P. Yuan, New parton distribution functions from a global analysis of quantum chromodynamics, Phys. Rev. D 93, 033006 (2016).

[34] S. Alekhin, J. Blümlein, and S. Moch, Parton distribution functions and benchmark cross sections at NNLO, Phys. Rev. D 86, 054009 (2012).

[35] L. A. Harland-Lang, A. D. Martin, P. Motylinski, and R. S. Thorne, Parton distributions in the LHC era: MMHT 2014 PDFs, Eur. Phys. J. C 75, 204 (2015).

[36] R. D. Ball et al. (NNPDF Collaboration), Parton distributions from high-precision collider data, Eur. Phys. J. C 77, 663 (2017).

[37] J. M. Campbell and R. Keith Ellis, Top-quark loop corrections in $Z+$ jet and $Z+2$ jet production, J. High Energy Phys. 01 (2017) 020.

[38] H. Ita and K. Ozeren, Colour decompositions of multi-quark one-loop QCD amplitudes, J. High Energy Phys. 02 (2012) 118.

[39] M. Cacciari, G. P. Salam, and G. Soyez, The anti- $k_{t}$ jet clustering algorithm, J. High Energy Phys. 04 (2008) 063.

[40] M. Cacciari, G. P. Salam, and G. Soyez, FastJet user manual, Eur. Phys. J. C 72, 1896 (2012).

[41] S. Catani, Y. L. Dokshitzer, M. H. Seymour, and B. R. Webber, Longitudinally invariant $k_{T}$ clustering algorithms for hadron hadron collisions, Nucl. Phys. B406, 187 (1993).

[42] G. P. Salam and G. Soyez, A practical Seedless Infrared-Safe Cone jet algorithm, J. High Energy Phys. 05 (2007) 086.

[43] D. Amati, A. Bassetto, M. Ciafaloni, G. Marchesini, and G. Veneziano, A treatment of hard processes sensitive to the infrared structure of QCD, Nucl. Phys. B173, 429 (1980).

[44] S. Catani, F. Krauss, R. Kuhn, and B. R. Webber, QCD matrix elements + parton showers, J. High Energy Phys. 11 (2001) 063.

[45] S. Catani, B. R. Webber, and G. Marchesini, QCD coherent branching and semiinclusive processes at large $\mathrm{x}$, Nucl. Phys. B349, 635 (1991).

[46] S. Catani, Y. L. Dokshitzer, M. Olsson, G. Turnock, and B. R. Webber, New clustering algorithm for multi-jet crosssections in e+ e- annihilation, Phys. Lett. B 269, 432 (1991).

[47] S. D. Ellis, R. Kleiss, and W. J. Stirling, W's, Z's and jets, Phys. Lett. 154B, 435 (1985); F. A. Berends, W. T. Giele, H. Kuijf, R. Kleiss, and W. J. Stirling, Multi-jet production in W, Z events at P anti-P colliders, Phys. Lett. B 224, 237 (1989); F. A. Berends, H. Kuijf, B. Tausk, and W. T. Giele, 
On the production of a $\mathrm{W}$ and jets at hadron colliders, Nucl. Phys. B357, 32 (1991); E. Abouzaid and H. J. Frisch, The ratio of $W+N$ jets to $Z^{0} / \gamma^{*}+N$ jets versus $N$ as a test precision test of the standard model, Phys. Rev. D 68, 033014 (2003).

[48] C. W. Bauer and B. O. Lange, Scale setting and resummation of logarithms in pp $\rightarrow \mathrm{V}+$ jets, arXiv:0905.4739.

[49] F. Krauss, A. Schälicke, S. Schumann, and G. Soff, Simulating $W / Z+$ jets production at the Tevatron, Phys. Rev. D 70, 114009 (2004).
[50] M. L. Mangano et al., Physics at a $100 \mathrm{TeV}$ pp collider: Standard Model processes, arXiv:1607.01831.

[51] D. Britzger et al. (fastNLO Collaboration), New features in version 2 of the fastNLO project, DOI: 10.3204/DESYPROC-2012-02/165.

[52] R. Boughezal, X. Liu, and F. Petriello, Phenomenology of the $\mathrm{Z}$ boson plus jet process at NNLO, Phys. Rev. D 94, 074015 (2016).

[53] C.-H. Kom and W. J. Stirling, Charge asymmetry in W + jets production at the LHC, Eur. Phys. J. C 69, 67 (2010). 